\title{
Texture Mapping with Hard Constraints Using Warping Scheme
}

\author{
Tong-Yee Lee, Member, IEEE, Shao-Wei Yen, and I-Cheng Yeh
}

\begin{abstract}
Texture mapping with positional constraints is an important and challenging problem in computer graphics. In this paper, we first present a theoretically robust, foldover-free 2D mesh warping algorithm. Then, we apply this warping algorithm to handle mapping texture onto 3D meshes with hard constraints. The proposed algorithm is experimentally evaluated and compared with the state-of-the-art method for examples with more challenging constraints. These challenging constraints may lead to large distortions and foldovers. Experimental results show that the proposed scheme can generate more pleasing results and add fewer Steiner vertices on the $3 \mathrm{D}$ mesh embedding.
\end{abstract}

Index Terms-Foldover, path-swapping, positional constraints, texture mapping, warping.

\section{INTRODUCTION}

$\mathrm{T}$ EXTURE mapping technique usually assigns $(u, v)$ texture image coordinates to 3D mesh vertices. This assignment can be computed using mesh planar parameterization. Many other methods [2], [3], [4], [5], [9], [10], [12], [13], [17], [20], [23], [25] have also been proposed. A more challenging problem is to compute this assignment with a special correspondence between the mesh geometry and the texture. This problem is called constrained texture mapping [2], [4], [10], [12], [25]. The hard constraint states that the feature vertices on the parametric domain are exactly aligned to the user-specified positions. In this paper, two major contributions are listed below:

1. For a given $2 \mathrm{D}$ mesh, we propose a theoretically robust and foldover-free warping algorithm to align the positional constraints.

2. We extend this new warping algorithm to handle texture mapping with hard constraints. In contrast to the state-of-the-art method [10], this new constrained texture mapping can handle more challenging constraints with less danger of distortions. Furthermore, our method adds a fewer number of Steiner vertices on the $3 \mathrm{D}$ mesh embedding.

The rest of the paper is organized as follows: Section 2 describes the most related work. We first present a foldover-free 2D mesh warping algorithm in Section 3 and then introduce our application to the constrained texture mapping in Section 4. Section 5 demonstrates the experimental results and gives discussion. Our conclusions and future work are given in Section 6.

- The authors are with the Computer Graphics Group/Visual System Laboratory, Department of Computer Science and Information Engineering, National Cheng-Kung University, No.1, Ta-Hsueh Road, Tainan 701, Taiwan, R.O.C.

E-mail: \{tonylee, ichenyen\}@mail.ncku.edu.tw,legends@csie.ncku.edu.tw.

Manuscript received 26 Oct. 2006; revised 28 June 2007; accepted 2 July 2007; published online 2 Aug. 2007.

Recommended for acceptance by C. Gotsman.

For information on obtaining reprints of this article, please send e-mail to: tvcg@computer.org, and reference IEEECS Log Number TVCG-0194-1006.

Digital Object Identifier no. 10.1109/TVCG.2007.70432.

$1077-2626 / 08 / \$ 25.00$ C 2008 IEEE

\section{ReLATEd WORK}

Several methods in [1], [2], [4], [6], [10], [11], [12], [19], [21], [25] have addressed the problem of parameterization under constraints, and several applications are explored including morphing, compatible parameterization, remeshing, and constrained texture mapping. Desbrun et al. [2] and Lévy [12] solved constraints using Lagrange multipliers and a least square system, respectively. However, both methods failed to guarantee a bijective embedding. On the other hand, Lee and Huang [14] used RBF to warp embedding for aligning features. However, this method can create foldovers. Eckstein et al. [4] used a constrained simplification to align the constraints and reconstructed simplified vertices without foldovers by adding Steiner vertices. Fujimura and Makarov [7] presented a 2D image warping method with several constraints. Their approach repeatedly used the Delaunay triangulation and edge swaps to avoid foldovers. The examples presented in [4] and [7] were simple; so, it is not clear how these methods were able to handle more complicated constraints. In addition, Zöckler et al. [26] also used [7] to handle constraints in 3D morphing. However, the authors mentioned that this approach could potentially create foldovers when the positions of the corresponding features are very distinct between two embeddings. Kraevoy et al. [10] described a state-of-the-art matchmaker algorithm for solving constraints in texture mapping. However, this algorithm may fail because it does not consider consistent neighbor ordering as mentioned in [21]. This greedy path-matching approach fails to handle challenging constraints well, and some examples will be shown in Section 5.

Many methods for surface parameterization with constraints have been introduced to embed a 3D surface onto a simpler intermediate domain. Alexa [1] suggested a computationally intensive relaxation-based approach to align feature vertices between two spherical parameterizations. This algorithm can potentially fail for a large number of features. Later, Lin et al. [16] used edge swaps to solve this problem on spherical embeddings. However, the edge swaps can damage the geometric surface. Praun et al. [19] consistently parameterized a set of genus- 0 meshes into a user-specified simplicial complex. However, this approach potentially produces swirling paths, thereby increasing the 


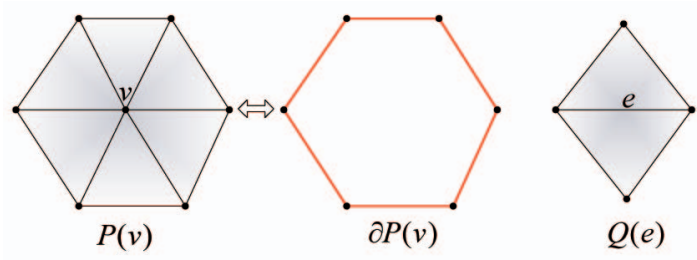

Fig. 1. $P(v)$ : the union of triangles adjacent to $v \cdot \partial P(v)$ : The boundary of $P(v) . Q(e)$ : the enclosing quadrilateral of $e$.

distortion. Later, Schreiner et al. [21] and Kraevoy and Sheffer [11] extended [10] and [19] to consistently parameterize models with the same genus. Recently, Lee et al. [15] proposed a method to consistently parameterize models with different genus on the spherical domain. In this method, the CSG operation potentially damages the surface, in particular, for areas near holes.

\section{Foldover-Free 2D Triangle Mesh Warping}

In this section, we will present a foldover-free 2D mesh warping method to satisfy positional constraints. This method uses edge swap operations to prevent triangles from folding over. The basic idea behind the proposed method is simple and well known. However, we will show that the edge swaps must be properly arranged; otherwise, they cannot solve foldovers. To the best of our knowledge, this is the first theoretically robust algorithm for solving foldovers that uses edge swaps only. Our approach, meanwhile, can detect foldovers in advance and determine where the edge swaps should be executed to resolve potential triangle foldovers.

\subsection{Definitions}

We consider a topologically disklike $2 \mathrm{D}$ triangular mesh $T$ with a convex boundary. The following notations are used to describe our warping algorithm:

- $\quad v$. An interior vertex of $T$.

- $\quad e$. An edge of $T$, and let $\overleftrightarrow{e}$ denote a line containing $e$.

- $\quad P(v)$. The union of triangles adjacent to $v$ (see Fig. 1).

- $\quad \partial P(v)$. The boundary (edges) of $P(v)$.

- $Q(e)$. The quadrilateral formed by the two adjacent triangles sharing an edge $e . Q(e)$ is convex if none of its internal angles is greater than $\pi$. An edge $e$ can be swapped if $Q(e)$ is convex.
- $\quad \tilde{v}$. A positional constraint for $v$ to align, and $\tilde{v}$ is inside triangular mesh $T$.

- $\overrightarrow{v_{m}}$. A moving direction defined as a ray starting from $v$ and passing through $\tilde{v}$, that is, $\overrightarrow{v_{m}}=\vec{v}$.

- $v_{\alpha}$. The invalid point defined as the following equation:

$$
v_{\alpha}=\arg \min _{p \in K}\|\overline{v p}\|
$$

where

$$
\begin{aligned}
K=\left\{x \mid x \text { is the intersection of } \overrightarrow{v_{m}}\right. \text { and } \\
\\
\stackrel{\leftrightarrow}{e}, \forall e \in \partial P(v)\} .
\end{aligned}
$$

- $\quad E_{\alpha}(v)$. An edge set defined as

$$
E_{\alpha}(v)=\left\{e \mid e \in \partial P(v) \text {, and } \overleftrightarrow{e} \text { contains } v_{\alpha}\right\} .
$$

Our foldover-free warping algorithm gradually moves $v$ along $\overrightarrow{v_{m}}$ to align $\tilde{v}$. If $\|\overrightarrow{v \tilde{v}}\| \geq\left\|\overrightarrow{v v_{\alpha}}\right\|$, we need to move $v$ to $v_{\alpha}$ first by appropriate edge-swaps and then continue moving it to $\tilde{v}$ gradually. The mesh therefore becomes warped. Fig. 2 shows three different possibilities of $\left.v_{\alpha}: 1\right) v_{\alpha}$ is on $E_{\alpha}(v)$, except the vertices of $\left.E_{\alpha}(v), 2\right) v_{\alpha}$ is not on $E_{\alpha}(v)$, and 3) $v_{\alpha}$ is on any vertex of $E_{\alpha}(v)$. We will show that $v$ can move to $v_{\alpha}$ without creating foldovers under the conditions of 1) and 2). If $v_{\alpha}$ is on any vertex of $E_{\alpha}(v)$, we will also show that this special case can be resolved such that $v$ can continue moving to $v_{\alpha}$ along $\overrightarrow{v_{m}}$.

\subsection{Theoretical Analysis and Algorithm}

In this section, the following theoretical analysis is given, and the results of analysis are used to derive our foldoverfree warping algorithm.

\subsubsection{Theoretical Analysis}

Lemma 1. If $\left|E_{\alpha}(v)\right|=1$ and $v_{\alpha}$ is on $E_{\alpha}(v)$, $v$ can move to $v_{\alpha}$ along $\overrightarrow{v_{m}}$ without flipping triangles after an edge swap operation.

Proof. $v_{\alpha}$ is not on any vertex of $E_{\alpha}$ because $\left|E_{\alpha}(v)\right|=1$. Let $E_{\alpha}(v)=e$. Then, we show that $e$ can be swapped before $v$ moves to $v_{\alpha}$ along $\overrightarrow{v_{m}}$. If $Q(e)$ is convex, then $e$ can be swapped. If $Q(e)$ is not convex, the following statements show that $e$ can still be swapped. In Fig. 3a, let $e=\overline{p_{1} p_{3}}$ and $v p_{1} p_{2} p_{3}$ be the quadrilateral $Q(e)$. Both interior angles

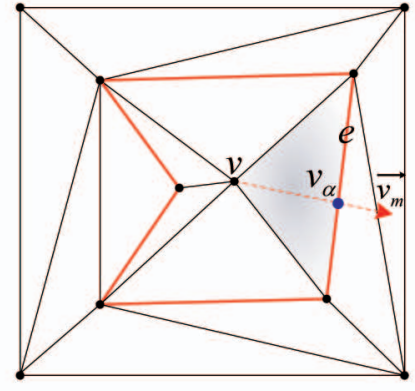

(a)

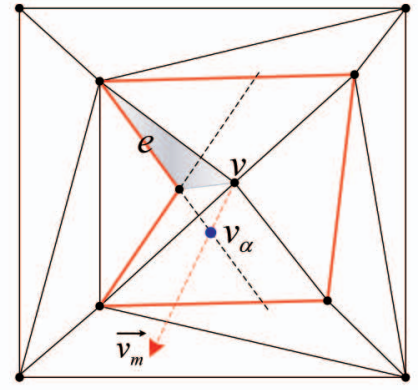

(b)

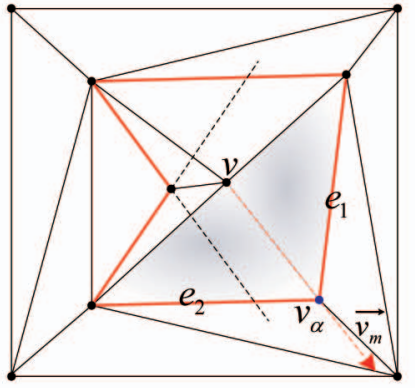

(c)

Fig. 2. $\partial P(v)$ consists of red edges. When $v$ moves to $v_{\alpha}$, each shaded triangle becomes degenerated, that is, folded over. (a) $E_{\alpha}(v)=\{e\}$. (b) $E_{\alpha}(v)=\{e\}$. (c) $E_{\alpha}(v)=\left\{e_{1}, e_{2}\right\}$. 


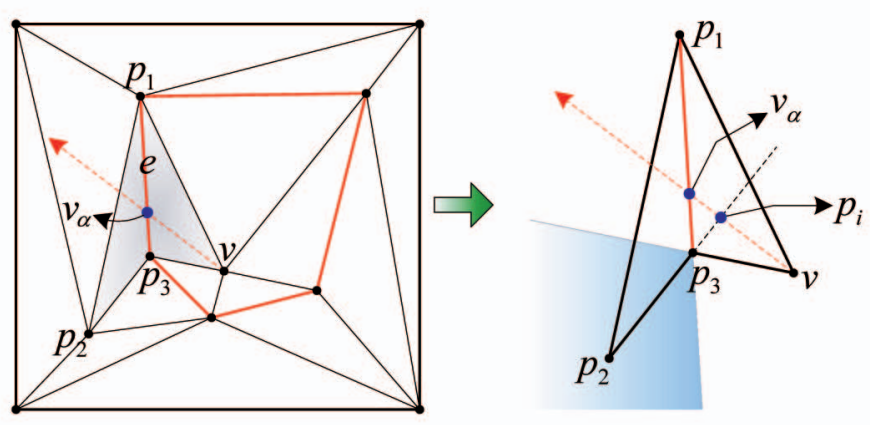

(a)

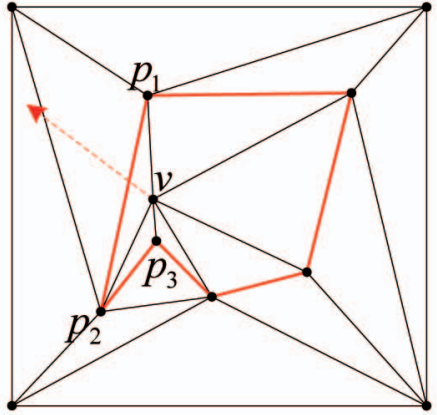

(b)

Fig. 3. (a) $E_{\alpha}(v)=\{e\}$. When $v$ moves to the open interval $\left(p_{i}, v_{\alpha}\right), Q(e)$ becomes convex and $e$ (that is, $\left.\overline{p_{1} p_{3}}\right)$ can be swapped. (b) Edge $e$ is swapped, and $v$ moves to $v_{\alpha}$.

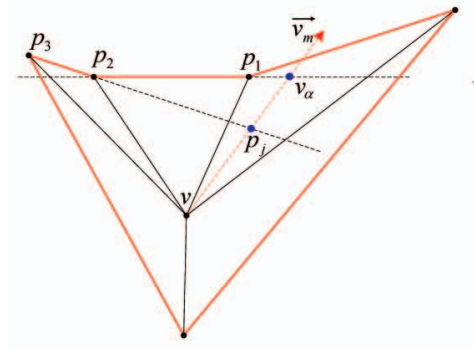

(a)

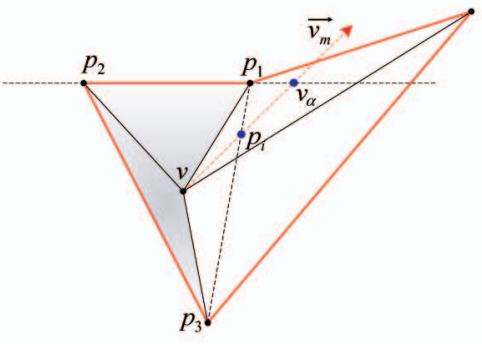

(b)

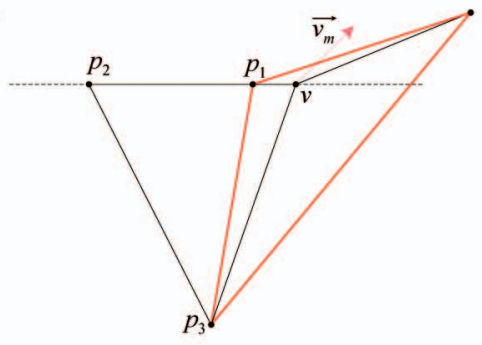

(c)

Fig. 4. (a) $\angle p_{1} p_{2} p_{3}>\pi$ in $Q\left(\overline{v p_{2}}\right)$. (b) $\angle p_{3} v p_{1} \geq \pi$ in $Q\left(\overline{v p_{2}}\right)$. (c) $\overline{v p_{2}}$ is swapped, and $v$ moves to $v_{\alpha}$.

$\angle p_{3} v p_{1}$ and $\angle p_{1} p_{2} p_{3}$ in $Q(e)$ are less than $\pi$ since they are the internal angles of triangles in 2D triangular mesh $T$. Without loss of generality, let $\angle p_{2} p_{3} v$ in $Q(e)$ be greater than or equal to $\pi$. Then, the vertex $p_{2}$ is in the shaded blue region defined by the intersection of two half-planes. These two half-planes are formed by $\overleftrightarrow{p_{1} p_{3}}$ and $\overleftrightarrow{p_{3} v}$, respectively, as shown in Fig. 3a. The intersection point $p_{i}$ of two lines $\overleftrightarrow{p_{2} p_{3}}$ and $\overleftrightarrow{v v_{\alpha}}$ is on the half-closed interval $\left[v, v_{\alpha}\right)$. If $v$ moves to the open interval $\left(p_{i}, v_{\alpha}\right), \angle p_{2} p_{3} v$ is less than $\pi$. Then, the edge $e$ can be swapped.

Since $\left|E_{\alpha}(v)\right|=1$, only the triangle $\Delta v p_{1} p_{3}$ containing $e$ is degenerated when $v$ moves to $v_{\alpha}$, as shown in Fig. 3a. After $e$ is swapped, the possibly degenerated triangle $\Delta v p_{1} p_{3}$ is removed and $\Delta v p_{1} p_{2}$, as well as $\Delta v p_{2} p_{3}$ are created. Thereafter, $\Delta v p_{1} p_{2}$ and $\Delta v p_{2} p_{3}$ are not flipped as $v$ moves to $v_{\alpha}$, as shown in Fig. $3 \mathrm{~b}$. Our result follows. $\square$

Lemma 2. If $\left|E_{\alpha}(v)\right|=1$ and $v_{\alpha}$ is not on $E_{\alpha}(v)$, $v$ can move to $v_{\alpha}$ along $\overrightarrow{v_{m}}$ without flipping triangles after an edge swap operation.

Proof. Let $E_{\alpha}(v)=\left\{\overline{p_{1} p_{2}}\right\} \cdot v_{\alpha}$ is on $\overleftrightarrow{p_{1} p_{2}}$ but not on $\overline{p_{1} p_{2}}$. Without loss of generality, we assume that $p_{2}$ is farther than $p_{1}$ from $v_{\alpha}$. Let $p_{3}$ be a vertex adjacent to both $v$ and $p_{2}$ such that $v p_{1} p_{2} p_{3}$ is $Q\left(\overline{v p_{2}}\right)$, as shown in Fig. $4 \mathrm{a}$ and $4 \mathrm{~b}$. The $\angle p_{1} p_{2} p_{3}$ in $Q\left(\overline{v p_{2}}\right)$ is not equal to $\pi$ since $\left|E_{\alpha}(v)\right|=1$. Let $p_{j}$ be the intersection of $\overrightarrow{v_{m}}$ and ${\overleftrightarrow{p_{2} p_{3}}}_{3}$. If $\angle p_{1} p_{2} p_{3}$ in $Q\left(\overline{v p_{2}}\right)$ is greater than $\pi, v_{\alpha}$ is farther than $p_{j}$ from $v$, as shown in Fig. 4a. This is a contradiction to the definition of $v_{\alpha}$. Then, we know that the vertex $p_{2}$ is convex for $Q\left(\overline{v p_{2}}\right)$.

Next, we show that the edge $\overline{v p_{2}}$ can be swapped before $v$ moves to $v_{\alpha}$. If the $Q\left(\overline{v p_{2}}\right)$ is not convex, we will show that $\overline{v p_{2}}$ can be still swapped. The $\angle v p_{1} p_{2}$ and $\angle p_{2} p_{3} v$ in $Q\left(\overline{v p_{2}}\right)$ are less than $\pi$ since they are the internal angles of triangles in a $2 \mathrm{D}$ triangular mesh $T$, and $\angle p_{1} p_{2} p_{3}$ in $Q\left(\overline{v p_{2}}\right)$ is also less than $\pi$ because $p_{2}$ is convex for $Q\left(\overline{v p_{2}}\right)$. Then, let $\angle p_{3} v p_{1} \geq \pi$ in $Q\left(\overline{v p_{2}}\right)$, as shown in Fig. $4 \mathrm{~b}$. The intersection point $p_{i}$ of $\overrightarrow{v_{m}}$ and $\overleftrightarrow{p_{1} p_{3}}$ is on the half-closed interval $\left[v, v_{\alpha}\right)$ since $\angle p_{3} v p_{1} \geq \pi$. If $v$ moves to the open interval $\left(p_{i}, v_{\alpha}\right)$, $\angle p_{3} v p_{1}<\pi$. Then, edge $\overline{v p_{2}}$ can be swapped.

Since $\left|E_{\alpha}(v)\right|=1$, only the triangle $\Delta v p_{1} p_{2}$ containing $\overline{p_{1} p_{2}}$ is degenerated when $v$ moves to $v_{\alpha}$, as shown in Fig. $4 \mathrm{~b}$. After $\overline{v p_{2}}$ is swapped, the possibly degenerated triangle $\Delta v p_{1} p_{2}$ is removed and $\Delta v p_{1} p_{3}$, as well as $\Delta p_{1} p_{2} p_{3}$, are created. Thereafter, $\Delta v p_{1} p_{3}$ and $\Delta p_{1} p_{2} p_{3}$ are not flipped as $v$ moves to $v_{\alpha}$. Our result follows.

Lemma 3. If $\left|E_{\alpha}(v)\right| \geq 1$ and $v_{\alpha}$ is not on $E_{\alpha}(v)$, $v$ can move to $v_{\alpha}$ along $\overrightarrow{v_{m}}$ without flipping triangles after a set of edge swap operations.

Proof. There are one or more connected components in $E_{\alpha}(v)$, as shown in Fig. 5. For each connected component $C$ of $E_{\alpha}(v)$, we will show that the adjacent triangle of each edge in $C$ can be removed before $v$ moves to $v_{\alpha}$, and then, no triangles are flipped when $v$ moves to $v_{\alpha}$. We prove this by induction on $|C|$ in the following:

Basis Step. $|C|=1$, let $C=\left\{\overline{q_{a} q_{b}}\right\}$ and follow the similar procedure of Lemma 2 , that is, let $\overline{q_{a} q_{b}}$ be $\overline{p_{1} p_{2}}$, as shown in Fig. 4 . We can find that the triangle $\Delta v q_{a} q_{b}$ containing $\overline{q_{a} q_{b}}$ can be removed and that the triangles created by the swap of $\overline{v q_{b}}$ are not degenerated when $v$ moves to $v_{\alpha}$.

Inductive Step. We assume that the statement is true while $|C|=k$. Now, consider $|C|=k+1$. First, all edges of $C$ are collinear since $C \subseteq E_{\alpha}$ and $C$ is connected. Let $\overline{q_{s} q_{t}} \in C$. Without loss of generality, $\overline{q_{s} q_{t}}$ is the farthest 


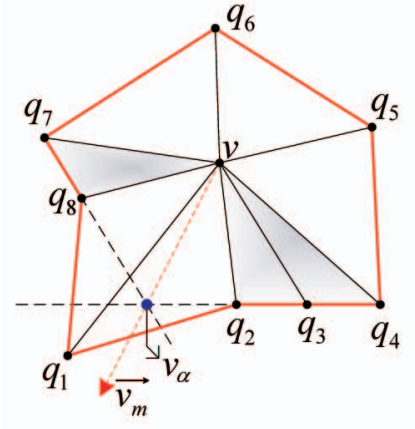

(a)

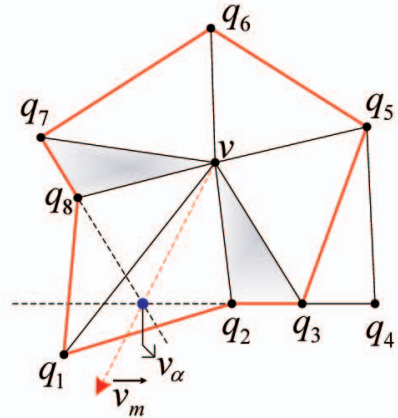

(b)

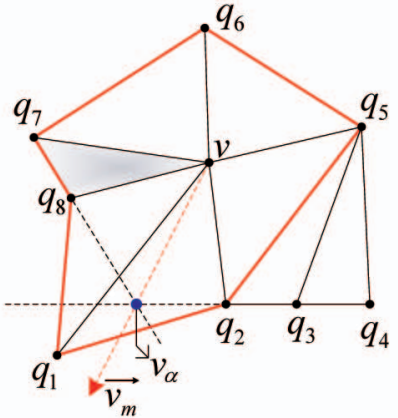

(c)

Fig. 5. Shaded triangles are degenerated when $v$ moves to $v_{\alpha}$. (a) $E_{\alpha}(v)=\left\{\overline{q_{2} q_{3}}, \overline{q_{3} q_{4}}, \overline{q_{7} q_{8}}\right\}$. The connected components of $E_{\alpha}(v)$ are $C_{1}=$ $\left\{\overline{q_{2} q_{3}}, \overline{q_{3} q_{4}}\right\}$ and $C_{2}=\left\{\overline{q_{7} q_{8}}\right\}$. Before, $v$ moves to $v_{\alpha}$, we can swap $\overline{v q_{4}}$ to remove $\Delta v q_{3} q_{4}$, as shown in (b), and then swap $\overline{v q_{3}}$ to remove $\Delta v q_{2} q_{3}$, as shown in (c).

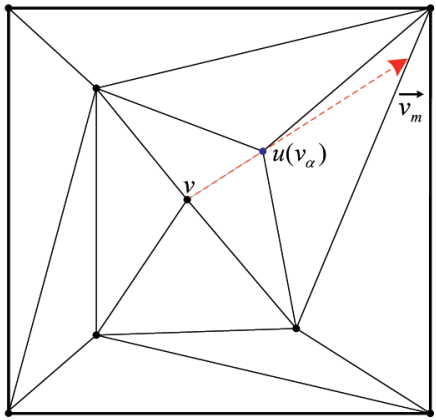

(a)

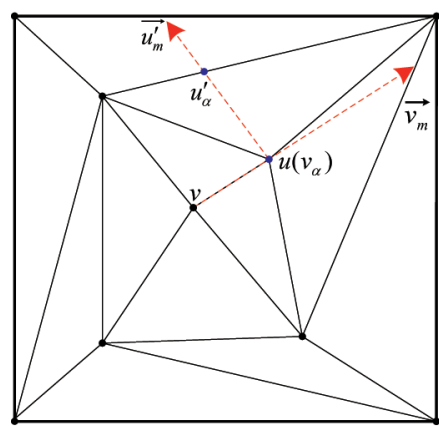

(b)

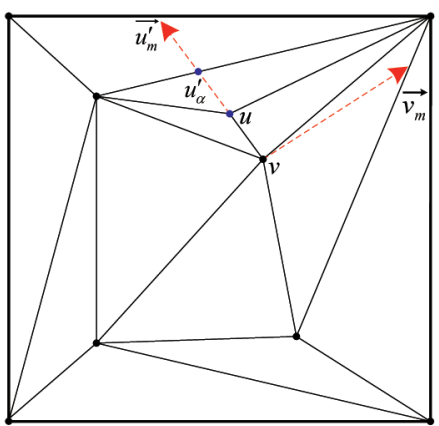

(c)

Fig. 6. (a) $v_{\alpha}$ is on a vertex $u$ of $E_{\alpha}(v)$. $u$ can find a direction $\overrightarrow{u_{m}^{\prime}}$ in (b). Then, $u$ can move to the interval $\left(u, u_{\alpha}^{\prime}\right)$ and $v$ can move to $v_{\alpha}$ in (c).

element of $C$ from $v_{\alpha}$, and $q_{t}$ is farther than $q_{s}$ from $v_{\alpha}$. If we follow the similar procedure as that used in Lemma 2, that is, let $\overline{q_{s} q_{t}}$ be $\overline{p_{1} p_{2}}$, as shown in Fig. 4 . The triangle $\Delta v q_{s} q_{t}$ containing $\overline{q_{s} q_{t}}$ can be removed, and the new triangles created by edge swap of $\overline{v q_{t}}$ are not degenerated when $v$ moves to $v_{\alpha}$ and then $|C|=k$. Using the induction hypothesis, the adjacent triangle of each edge in $C$ can be removed, as shown in Fig. 5. Our result follows.

Lemma 4. If $\left|E_{\alpha}(v)\right| \geq 1$ and $v_{\alpha}$ is on $E_{\alpha}(v)$, but not on the vertices of $E_{\alpha}(v), v$ can move to $v_{\alpha}$ along $\overrightarrow{v_{m}}$ without flipping triangles after a set of edge swap operations.

Proof. We will show that the adjacent triangle of each edge in $E_{\alpha}(v)$ can be removed before $v$ moves to $v_{\alpha}$ and that no triangles are flipped when $v$ moves to $v_{\alpha}$. We classify the edges of $E_{\alpha}(v)$ into two sets $B_{1}$ and $B_{2}$.

1. $B_{1}=\left\{\overline{q_{i} q_{j}} \mid \overline{q_{i} q_{j}} \in E_{\alpha}(v)\right.$ and $v_{\alpha}$ is on $\left.\overline{q_{i} q_{j}}\right\}$. Then, $\left|B_{1}\right|=1$ because $v_{\alpha}$ can be on one edge of $E_{\alpha}(v)$ at most. Let $B_{1}=\left\{\overline{q_{m} q_{n}}\right\}$, we can follow the similar procedure as that used in Lemma 1, that is, let $\overline{q_{m} q_{n}}$ be the edge $e$, as shown in Fig. 3. Then, we can find that $\overline{q_{m} q_{n}}$ can be swapped to remove the triangle $\Delta v q_{m} q_{n}$ and that the new triangles created by the edge swap of $\overline{q_{m} q_{n}}$ are not degenerated when $v$ moves to $v_{\alpha}$.

2. $B_{2}=\left\{\overline{q_{i} q_{j}} \mid \overline{q_{i} q_{j}} \in E_{\alpha}(v)\right.$ and $v_{\alpha}$ is not on $\left.\overline{q_{i} q_{j}}\right\}$. By following the similar procedure of Lemma 3, the adjacent triangle of each edge in $B_{2}$ can be removed, and the new triangles are not degenerated when $v$ moves to $v_{\alpha}$. Our result follows.

Lemma 5. If $v_{\alpha}$ is on a vertex $u$ of $E_{\alpha}(v), v$ can move to $v_{\alpha}$ along $\overrightarrow{v_{m}}$ without flipping triangles.

Proof. We first show that $u$ can be temporarily moved, such that $u$ is not on the direction of $\overrightarrow{v_{m}}$. We create a moving direction $\overrightarrow{u_{m}^{\prime}}$ for $u$ by randomly picking a direction that is not the same as $\overrightarrow{v_{m}}$ and $-\overrightarrow{v_{m}}$, as shown in Fig. 6a. Then, $u$ can find a temporary invalid point $u_{\alpha}^{\prime}$ by $\overline{u_{m}^{\prime}}$. If $u$ moves to the open interval $\left(u, u_{\alpha}^{\prime}\right), u$ is not on the direction of $\overrightarrow{v_{m}}$, and no triangles are flipped. Then, by Lemma 3 or Lemma $4, v$ can continue moving to $v_{\alpha}$, as shown in Fig. 6c. Our result follows.

In addition, Fig. $6 \mathrm{c}$ shows that $u$ can also move back to $v_{\alpha}$ along $-\overrightarrow{u_{m}^{\prime}}$ to restore its original position by Lemma 3 or Lemma 4 after $v$ leaves $v_{\alpha}$ along $\overrightarrow{v_{m}}$.

We can state the following theorem from Lemma 3, Lemma 4, and Lemma 5.

Theorem 1. $v$ can move to the invalid point $v_{\alpha}$ along $\overrightarrow{v_{m}}$ without flipping triangles.

\subsubsection{Foldover-Free Warping Algorithm}

Now, we will describe a foldover-free 2D mesh warping algorithm in MeshWarp() based on the above analysis. Iteratively, this algorithm moves each vertex $v$ along $\overrightarrow{v_{m}}$ to $\tilde{v}$. If $v$ is required to move to $v_{\alpha}$, the algorithm executes edge 
swaps to remove possibly degenerated triangles. Then, $v$ moves to $v_{\alpha}$ using MoveToInvalidPoint $(v)$. The procedure MoveToInvalidPoint() is derived from Lemma 3, Lemma 4, and Lemma 5. Note that the StepSpeed in MeshWarp() is a user-defined constant to control the warping speed, and MinimalStep $(T)$ returns the minimal distance between each $\overline{v \tilde{v}}$ for all unaligned vertices. In our experiment, if the StepSpeed constant is set too large, the number of edge swaps would become bigger, and the large distortion would occur in base mesh. From our experimental experience, the value 0.1 is a good constant for the StepSpeed.

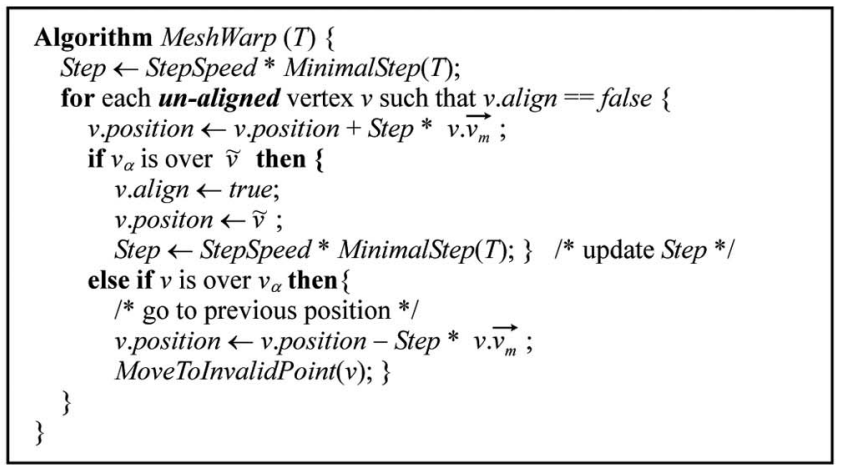

Procedure MoveToInvalidPoint(v)

\{

if $v_{\alpha}$ is not on any vertex of $E_{\alpha}(v)$ then

The edges of $E_{\alpha}(v)$ are classified into two sets $B_{1}$ and $B_{2}$ as in Lemma 4.

if $B_{1}$ is not empty then \{

Let $B_{1}=\left\{\overline{q_{m} q_{n}}\right\}$ and $Q\left(\overline{q_{m} q_{n}}\right)$ is $v q_{m} q_{o} q_{n}$

if $\angle v q_{m} q_{0} \geq \pi$ then $\quad / *$ internal angle of $Q\left(\overline{q_{m} q_{n}}\right) * /$

Compute the intersection $p_{i}$ of $\overleftrightarrow{q_{o} q_{m}}$ and $\overleftrightarrow{v v_{\alpha}}$;

else if $\angle v q_{n} q_{0} \geq \pi$ then

Compute the intersection $p_{i}$ of $\overleftrightarrow{q_{o} q_{n}}$ and $\overleftrightarrow{v_{\alpha}}$;

else then $p_{i} \leftarrow v$.position;

v.position $\leftarrow 0.5 *\left(p_{i}+v_{\alpha}\right) ; / * v$ moves to $\left(p_{i}, v_{\alpha}\right) * /$ $\left.\operatorname{EdgeSwap}\left(\overline{q_{m} q_{n}}\right) ;\right\}$

if $B_{2}$ is not empty then \{

do

for each connected component $C \subseteq B_{2}\{$

Let $\overline{q_{s} q_{t}} \in C, \overline{q_{s} q_{t}}$ is the farthest element from $v_{\alpha}$ in $C$,

and $q_{t}$ is farther then $q_{s}$ from $v_{\alpha}$;

Let $Q\left(\overline{v q_{t}}\right)$ be $v q_{s} q_{t} q_{u}$;

if $\angle q_{u} v q_{s} \geq \pi$ then $\left\{/ *\right.$ internal angle of $Q\left(\overline{v q_{t}}\right) * /$

Compute the intersection $p_{i}$ of $\overleftrightarrow{q_{u} q_{s}}$ and $\overleftrightarrow{v_{\alpha}}$;

v.position $\leftarrow 0.5 *\left(p_{i}+v_{\alpha}\right)$;

$\operatorname{EdgeSwap}\left(\overline{v q_{t}}\right) ; / *$ eliminate one element of $\left.C * /\right\}$

i* for loop */

while $B_{2}$ is not empty ; /* while loop */ \}

$v$.position $\leftarrow v_{\alpha}$;

UpdateInvalidPoint $(v) ; \quad / *$ find the next $v_{\alpha}$ of $\left.v * /\right\}$

else if ( $v$ is on a vertex $u$ of $\left.E_{\alpha}(v)\right)$ then \{

create $\overrightarrow{u_{m}^{\prime}}$, and compute $u_{\alpha}^{\prime}$ by $\overrightarrow{u_{m}^{\prime}} ; / *$ (see Fig. 6) */

move $u$ to open interval $\left(u, u_{\alpha}^{\prime}\right)$;

move $v$ to $v_{\alpha} ; \quad$ * use Lemma 3 or Lemma $4 *$ /

UpdateInvalidPoint $(v)$;

$v$.position $\leftarrow 0.5 *\left(v+v_{\alpha}\right)$;

move $u$ to its original position ; /* use Lemma 3 or Lemma $4 * /$ \}

$$
\text { \} }
$$

The warping algorithm may possibly generate very skinny triangles that would cause a numerical error in computation. From our practice, it is usually due to a very small "Step" in MeshWarp(T). We avoid this problem by limiting the value of "Step" to not be smaller than a predefined threshold. In addition, before an edge swap, when $v$ is required to move to the open interval $\left(p_{i}, v_{\alpha}\right)$, the vertex $v$ is always moved to $0.5 *\left(p_{i}+v_{\alpha}\right)$ in MoveToInvalidPoint $(v)$. Such movement is also useful in preventing the generation of skinny triangles.

\section{Texture Mapping with Hard Constraints}

\subsection{Overview and Definitions}

We now extend our new warping algorithm to compute surface parameterization for texture mapping with hard constraints. Figs. 7a, 7b, 7c, 7d, 7e, 7f, and 7g show the overall stages. To present this application, we define some terminology used in the following sections. Let $M$ be the input triangle mesh and a set of positional correspondence between $M$ and texture specified at Figs. $7 \mathrm{a}$ and $7 \mathrm{~b} . P^{M}$ is the planar embedding of $M$ and can be obtained using any bijective planar parameterization. In our implementation, $P^{M}$ is computed with that in [24]. We also add a set of virtual points surrounding $P^{M}$, as suggested in [10], such that the boundary of $P^{M}$ is free to move in the parametric domain. Let $M+$ be the mesh $M$ with virtual points, and the embedding of $M+$ be $P^{M+}$, as shown in Fig. 7c. In $P^{M+}$, each feature vertex (red point at the stage in Fig. 7c) corresponds to a feature vertex of $M$ (red point in Fig. 7a). The feature vertex of $P^{M+}$ will be aligned to a user-specified position on a texture image (red points in Fig. $7 \mathrm{~b}$ ) using our warping algorithm in Figs. 7d, 7e, and 7f. Finally, in Fig. 7g, a variant of stretch metric [20] is used to smooth embedding for reducing distortion. Later in Section 5, we will experimentally compare our method in [10] and give a discussion with the related work.

\subsection{Constructing a Base Mesh}

We do not directly use the warping algorithm in Section 3 to match feature vertices of $P^{M+}$ since the edge swap operations in $P^{M+}$ will damage the geometry of mesh $M$. To avoid this problem, we warp a base mesh $B^{M+}$ instead of $P^{M+}$. A base mesh $B^{M+}$ is constructed from $P^{M+}$. An edge swap in $B^{M+}$ corresponds to a path swap in $P^{M+}$, such that a path swap does not change the geometry of mesh $M$. The construction of base mesh $B^{M+}$ is described as follows:

Let $G$ be a guiding mesh, which is used to construct the base mesh. The vertex set of $G$ consists of feature vertices and virtual points from $P^{M+}$. Then, we can determine the connectivity of $G$ by the constrained Delaunay triangulation [22]. An example of $G$ is illustrated as a red-color-edge mesh in Fig. 7d. Each edge of $G$ guides us to compute a path on $P^{M+}$. We use the following steps to find such paths (the green paths shown in Fig. 7d).

1. For each inner edge $E_{G} \in G$, we first determine a closed region in order to locally compute its corresponding path on $P^{M+}$. Initially, the boundary of the closed region is formed by the enclosing quadrilateral of $E_{G}$, as shown in Fig. 8a. If the corresponding path of $E_{G}$ is found, $E_{G}$ is replaced by this path. For example, a guiding edge $\overline{f_{3} f_{4}}$ is replaced by the green path from $f_{3}$ to $f_{4}$, as shown in Fig. $8 \mathrm{~b}$. 


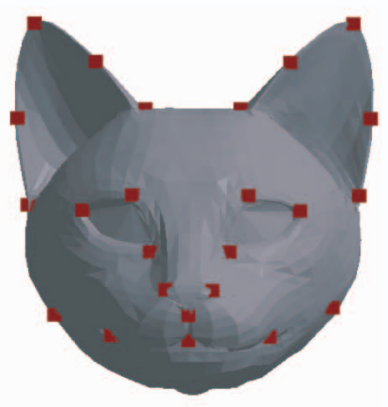

(a)

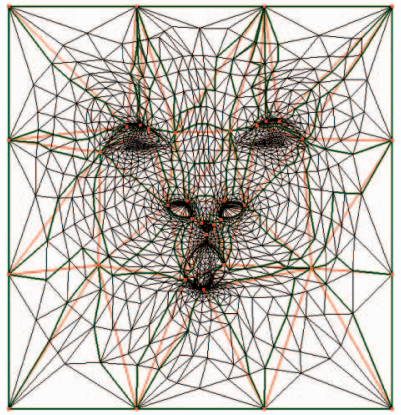

(d)

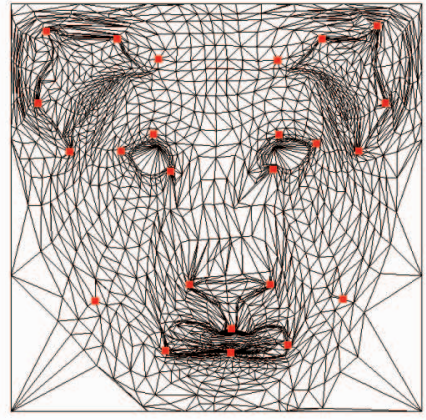

(g)

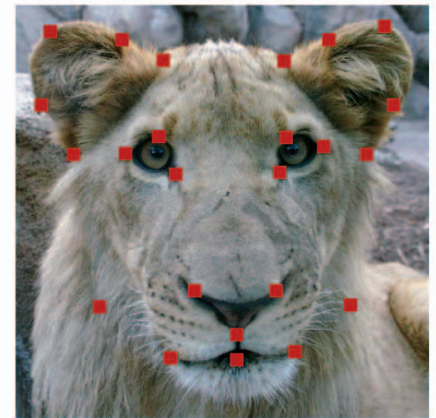

(b)

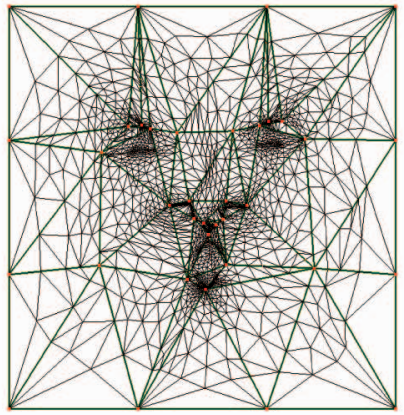

(e)

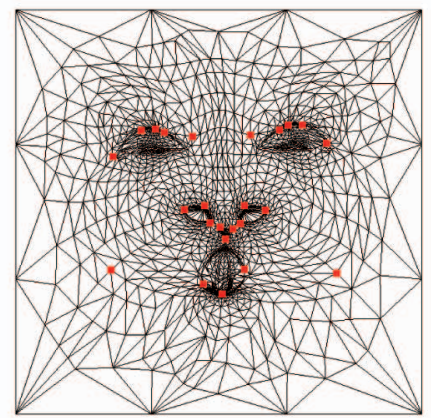

(c)

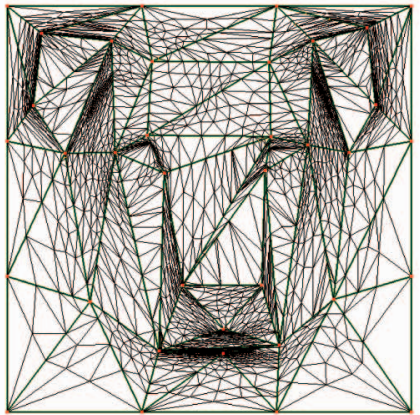

(f)

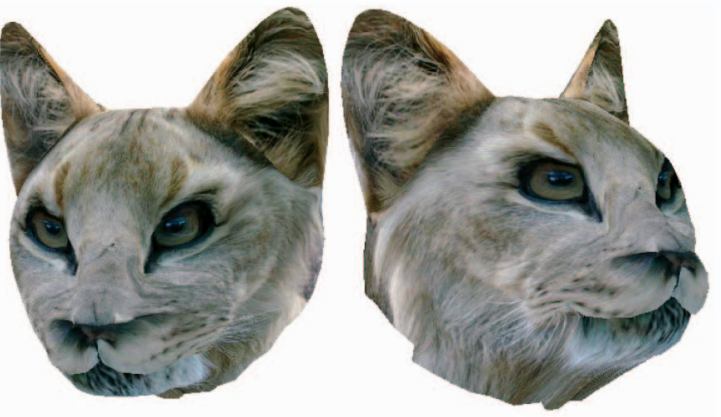

(h)

Fig. 7. (a) and (b) Input triangle mesh $M$ and 2D texture. (c) The planar embedding of $M$ with virtual boundary. (d), (e), and (f) Warping algorithm is used to align constraints. $(\mathrm{g})$ The resulting embedding is further smoothed. (h) The resulting textured model.

2. Next, if there exists some edge $\overline{v_{m} v_{n}}$ in $P^{M+}$, such that $\overline{v_{m} v_{n}}$ divides the enclosing boundary of $E_{G}$ into two disjoint subregions, then both end points of $\overline{v_{m} v_{n}}$

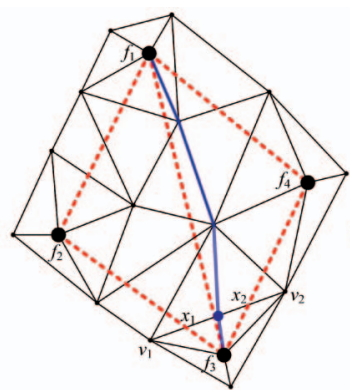

(a)

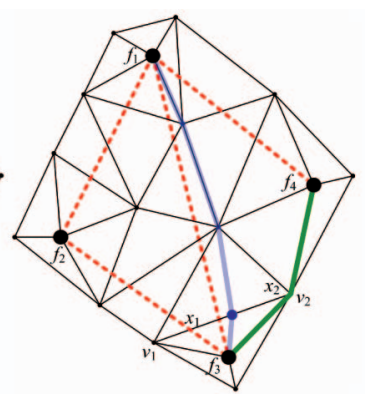

(b)
Fig. 8. $f_{i}$ are feature vertices, and red dotted lines are edges of $G$. A corresponding path (green) of $\overline{f_{3} f_{4}}$ was computed in (b). In (a) and (b), an edge $\overline{v_{1} v_{2}}$ of $P^{M+}$ divides the enclosing boundary of $\overline{f_{1} f_{3}}$ into two subregions. A Steiner vertex (blue) is added, and the corresponding path of $\overline{f_{1} f_{3}}$ is shown in light blue. can be either outside or are right on the enclosing boundary of $E_{G}$, for example, an edge $\overline{v_{1} v_{2}}$, as shown in Fig. 8. This edge $\overline{v_{m} v_{n}}$ potentially blocks the corresponding path of $E_{G}$. If $\overline{v_{m} v_{n}}$ is split by a Steiner vertex, the corresponding path of $E_{G}$ can be found. Therefore, we will add a Steiner vertex at the midpoint of $\overline{x_{m} x_{n}}$. The following rules are used to compute $x_{m}$ and $x_{n}$.

- If both end points of $\overline{v_{m} v_{n}}$ are outside the boundary of $E_{G}, x_{m}$ and $x_{n}$ become the intersection points of $\overline{v_{m} v_{n}}$ and the boundary of $E_{G}$, for example, $x_{1}$ and $x_{2}$ in Fig. 8a.

- If one end point $v_{m}$ of $\overline{v_{m} v_{n}}$ is outside the boundary of $E_{G}$, and the other $v_{n}$ is right on the boundary of $E_{G}, x_{m}$ becomes the intersection point of $\overline{v_{m} v_{n}}$ and the boundary of $E_{G}$, and $x_{n}$ is the same with $v_{n}$, for example, $x_{1}$ and $x_{2}$ in Fig. $8 \mathrm{~b}$.

- If both end points of $\overline{v_{m} v_{n}}$ are right on the boundary of $E_{G}, x_{m}$ can be $v_{m}$, and $x_{n}$ can be $v_{n}$. 


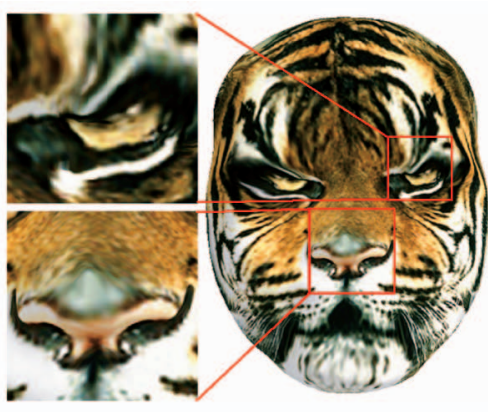

(a)

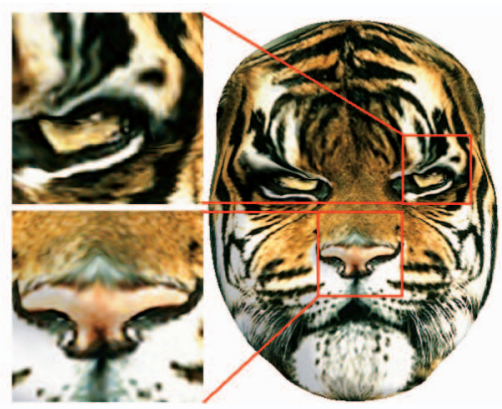

(b)

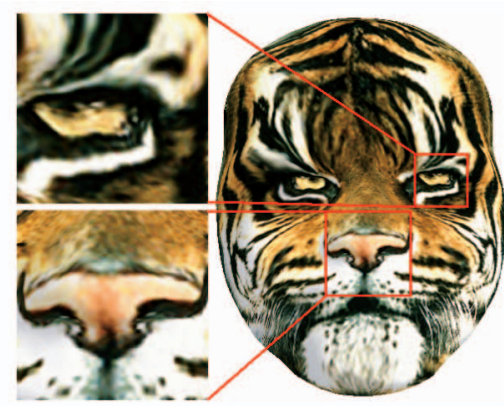

(c)

Fig. 9. Mesh smoothing using (a) [10], (b) the metric $\phi(t)$, and (c) the weighted metric $w \cdot \phi(t)$.

3. We can find the correspondent path of $E_{G}$ by Dijkstra's shortest path algorithm within the enclosing boundary of $E_{G}$. Only the vertices inside the enclosing boundary of $E_{G}$ can be joined to the shortest path computation.

In addition, for each boundary edge of $G$, its corresponding path consists of the boundary edges of $P^{M+}$. After constructing the corresponding path of each edge in $G, P^{M+}$ is then decomposed into a set of triangular patches, for example, in Fig. 7d, the boundary of each patch is drawn in green. Then, we can straighten the boundary of each patch in $P^{M+}$, and the base mesh $B^{M+}$ would therefore be created, as shown in Fig. 7e. We parameterize the vertices inside each patch by Tutte's method [24]. Then, the vertices inside each triangular patch are encoded by the barycentric coordinates with respect to the triangle nodes of $B^{M+}$. Note that before we straighten the boundary of each base triangle, there may be some vertices outside the corresponding base triangle. However, after 1) straightening the boundary of each triangle and 2) parameterizing the vertices inside each triangle patch using [24], the vertex inside the triangle patch is inside the corresponding triangle of base mesh, and the resulting barycentric coordinates become valid.

\subsection{Warping a Base Mesh}

To match constraints, we warp a base mesh $B^{M+}$ instead of an embedding $P^{M+}$. Each edge swap of $B^{M+}$ corresponds to a path swap since the edge of base mesh is actually a path on $P^{M+}$. Say, the edge $e$ of $B^{M+}$ to be swapped. First, we find the quadrilateral $Q(e)$ and reparameterize the inner vertices contained in $Q(e)$ using barycentric parameterization [24]. From Section 3, we know that $Q(e)$ can become convex before the edge swap. Therefore, the barycentric parameterization is always valid. Next, let the other diagonal of $Q(e)$ be a guiding edge to compute a new path $P$ for the swap of $e$. The new path $P$ is computed by Dijkstra's shortest path algorithm. An additional Steiner vertex will be added if there exists an edge in $P^{M+}$, such that its two end points are on the boundary of $Q(e)$. Once path $P$ is determined, it needs to be straightened, and two new triangular patches adjacent to $P$ are reparameterized. Therefore, the edge $e$ of $B^{M+}$ is swapped. Note that the inner vertices in each triangular patch of $B^{M+}$ are encoded by the barycentric coordinates. These vertices are extracted to compute a new path before an edge swap in $B^{M+}$, and the barycentric coordinates of these vertices need to be updated after an edge swap in $B^{M+}$.

\subsection{Smoothing the Embedding}

After aligning the constraints, the embedding is distorted. As suggested in [10], the postembedding smoothing will be useful in reducing distortion. Our smoothing procedure is a variant in [20] and is based on a restricted and iterative relaxation procedure. All feature vertices are restricted to be stationary, whereas the positions of other nonfeature vertices are iteratively adjusted. The adjustment aims at minimizing a stretch metric $\phi(t)$ for each triangle $t$ defined below:

$$
\begin{aligned}
& \phi(t)=\alpha_{\text {stretch }}^{2}+\beta_{\text {stretch }}^{2}, \\
& \text { where } \alpha_{\text {stretch }}=\left\{\begin{array}{l}
\Gamma, \Gamma \geq 1 \\
1 / \Gamma, \Gamma \leq 1
\end{array} \text { and } \beta_{\text {stretch }}=\left\{\begin{array}{l}
\gamma, \gamma \geq 1 \\
1 / \gamma, \gamma \leq 1
\end{array}\right.\right.
\end{aligned}
$$

$\Gamma$ and $\gamma$ are singular values derived from that in [20]. In $\phi(t)$, both enlargement $\Gamma$ and shrinkage $\gamma$ are treated with the same importance to measure the distortion of $t$. To further reduce the distortion for the neighborhood of the feature vertex, we use the weighted distortion metric $w \cdot \phi(t)$ instead of $\phi(t)$ to smoothen the embedding. The value of $w$ is computed by $1 / d_{f}^{2}$, where $d_{f}$ is the distance between the centroid of $t$ and the

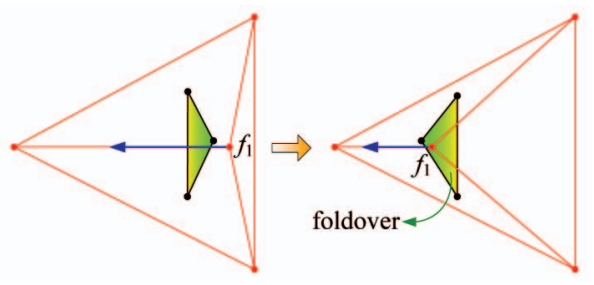

Fig. 10. Example of failure in Zöckler et al. [26]. The red nodes are feature vertices, and the black nodes are the other mesh vertices encoded by the barycentric coordinates. The shaded triangle is flipped when the feature $f_{1}$ continues moving. 


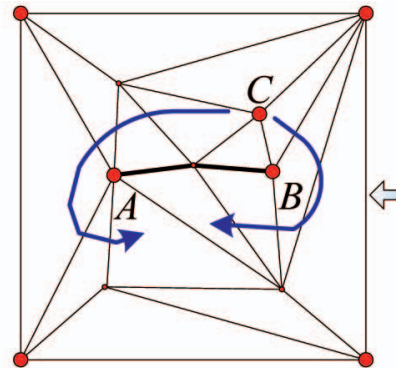

Parametric map $\left(P^{M+}\right)$

(a)

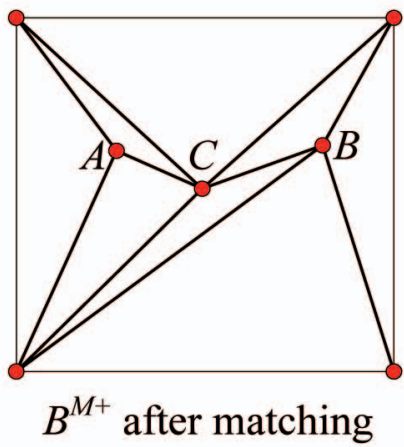

(c)

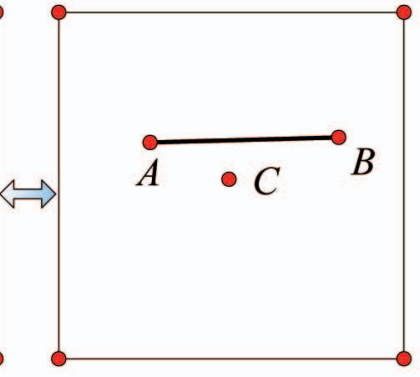

Texture

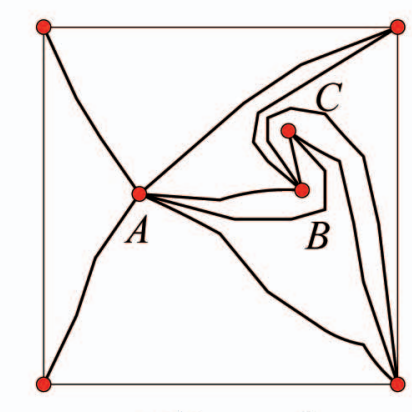

matching paths

(d)

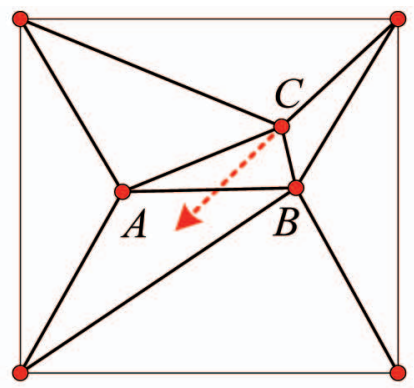

base mesh $B^{M+}$ of $P^{M^{+}}$

(b)

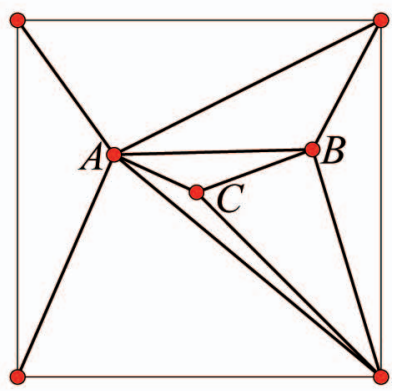

feature matching

(e)

Fig. 11. (a) Feature vertex $C$ is at the different side of path $A B$. (b) Path $A B$ does not prevent $C$ from moving since it can be swapped. (c) Feature matching in (b). (d) The matching paths using [10]. (e) Feature matching from that in (d). Note that the vertices inside each triangle patch are not shown in (b), (c), (d), and (e) for easy illustration.

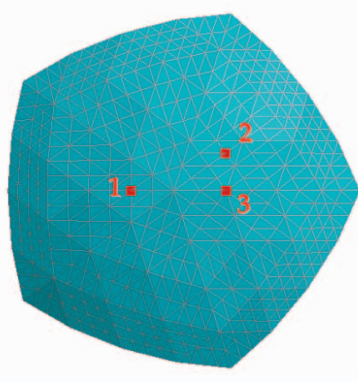

(a)

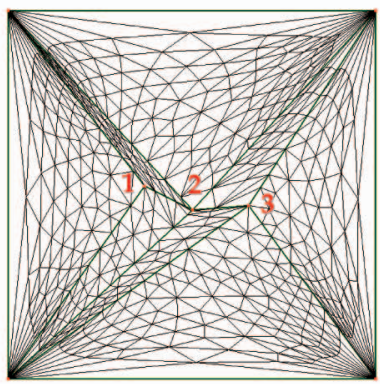

(e)

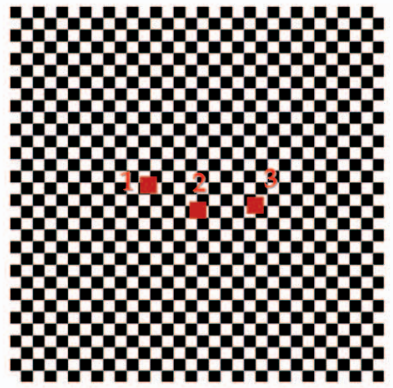

(b)

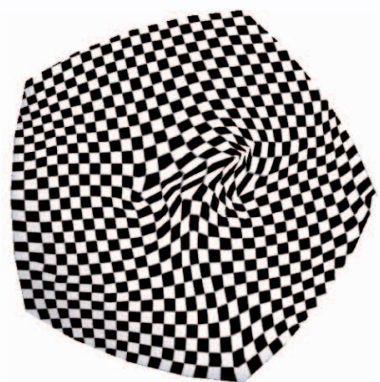

(f)

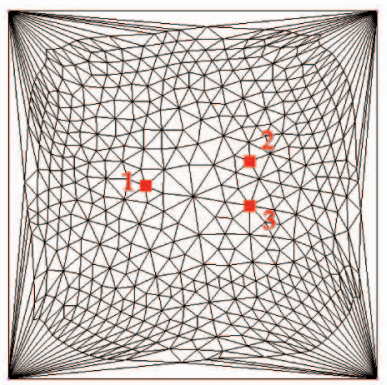

(c)

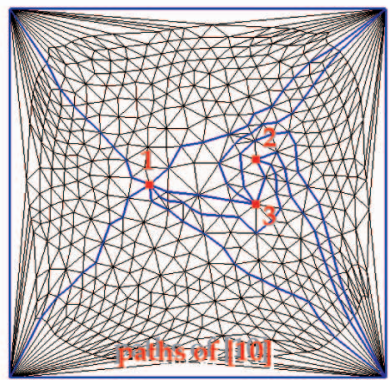

(g)

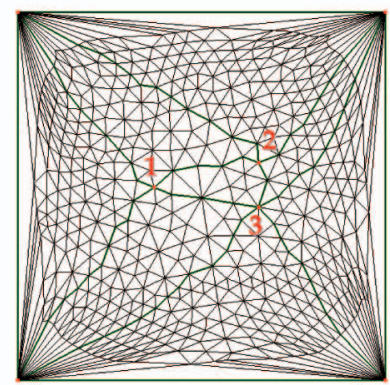

(d)

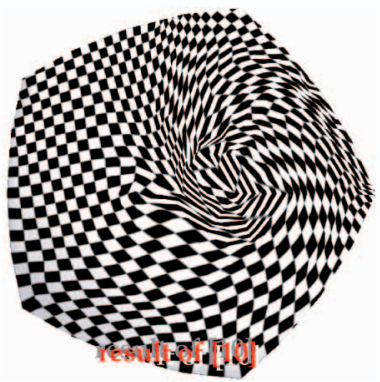

(h)

Fig. 12. (a) Input mesh $M$. (b) Input texture. (c) The embedding $P^{M+}$ of $M$. (d) The initial paths (green) of base mesh $B^{M+}$. (e) $B^{M+}$ after feature matching. (f) Texture mapping result after smoothing from (e). (g) The path matching using [10]. (h) Texture mapping result in [10].

nearest feature vertex of $t$. Fig. 9 shows an experimental comparison between [10] and our mesh smoothing method using $\phi(t)$ and $w \cdot \phi(t)$. The weighted metric $w \cdot \phi(t)$ performs the best in this example.

\section{EXPerimental Results AND Discussion}

\subsection{Results}

When corresponding features are very distinct, it is easy to yield large distortions and foldovers. We experimentally 


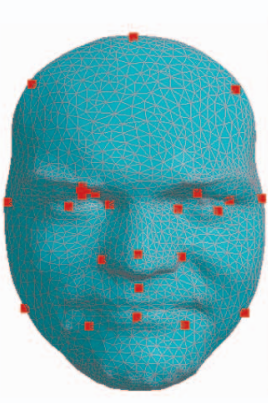

(a)

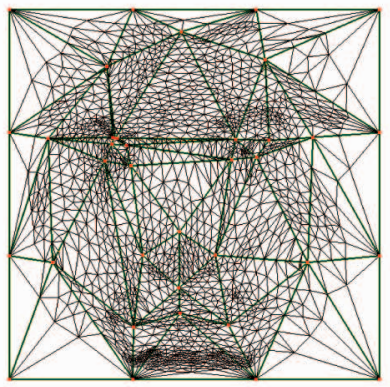

(e)

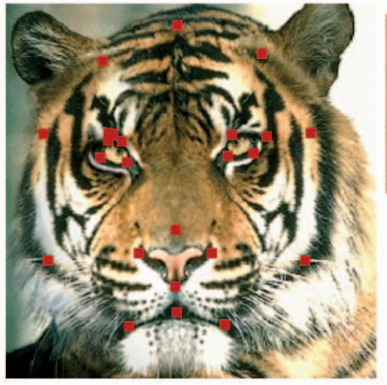

(b)

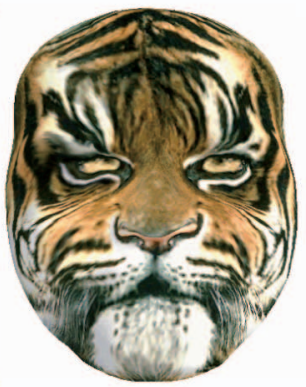

(f)

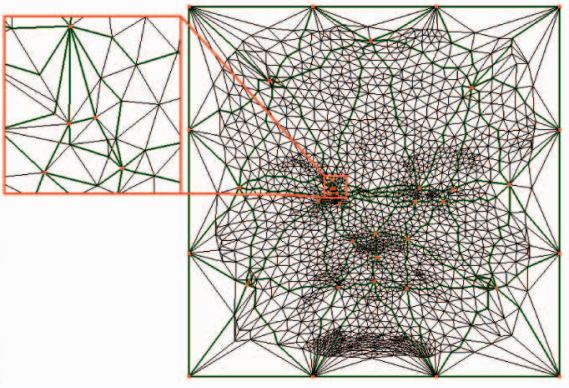

(c)

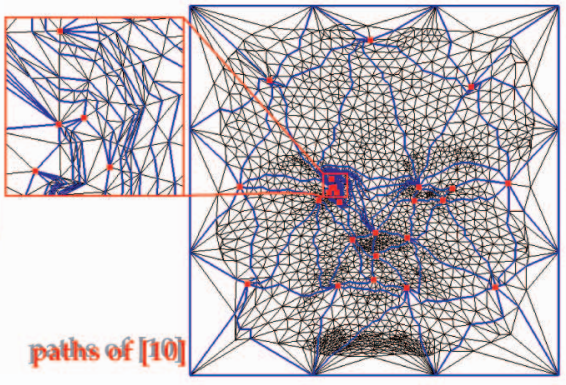

(g)

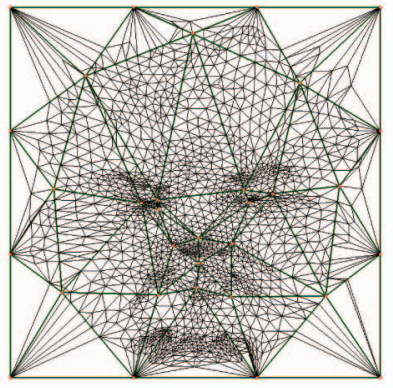

(d)

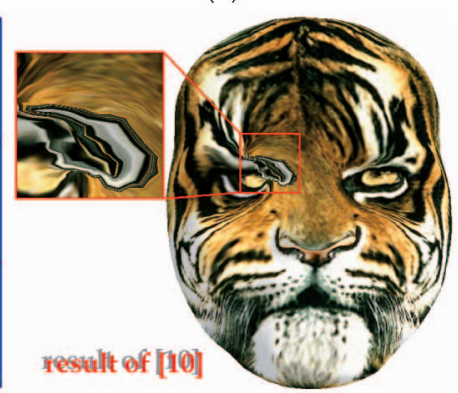

(h)

Fig. 13. (a) Input mesh $M$. (b) Input texture. (c) The embedding $P^{M+}$ of $M$ and the initial paths (green) of base mesh $B^{M+}$. (d) Initial base mesh $B^{M+}$. (e) $B^{M+}$ after feature matching. (f) Texture mapping result after smoothing from (e). (g) The path matching using [10]. (h) Texture mapping result in [10].

compare the proposed method and that in [10] for challenging examples as follows: The initial planar embedding for each example is computed by Tutte [24]. In Fig. 12, we design a special example, where the orientation of corresponding features is different between the texture in Fig. $12 \mathrm{~b}$ and embedding in Fig. 12c. As shown in Figs. 12g and $12 \mathrm{~h}$, the proposed method creates a much better result. In Fig. 12f, long swirls are created by their approach, and thus, a large distortion is made in Fig. 12h. In Figs. 12d and $12 \mathrm{e}$, our method simply swaps the edge $e_{13}$ and moves feature vertex $v_{2}$ to its positional constraint. Other two examples are evaluated, as shown in Figs. 13 and 14. In both examples, in [10], a number of Steiner vertices are added since many paths wind around some feature vertices, as shown in Figs. 13f and 14f. Figs. 13g and 14g show that our result is still better than Figs. 13h and 14h. Furthermore, we compare the number of Steiner vertices introduced by both methods. We add 0, 10, and 34 Steiner vertices for those in Figs. 12, 13, and 14, respectively. However, the number of Steiner vertices created by their approach is 12,368 , and 2,687 for Figs. 12, 13, and 14, respectively. In Figs. 12, 13, and 14, the texture mapping results in [10] are obtained after smoothing is applied by our proposed smoothing method. There are some other nice results created by our method, as shown in Figs. 15, 16, and 17. Kraevoy et al. [10] mentioned some challenging examples similar to those in Figs. 16 and 17. Our method can also handle these similar examples well. In Fig. 15, the cow example (Figs. 15c and $15 d)$ is a very challenging one due to lots of deformations in the horns. We show the final planar embeddings after smoothing for the cow model, especially near the horns.
Table 1 shows several statistics for our experiments. We implemented the proposed algorithm on an Intel Pentium 4, 2.4-GHz PC with 1 Gbyte of RAM. In our implementation, we also removed unnecessary Steiner vertices after mesh optimization, as suggested in [10]. For Figs. 15, 16, and 17, the texture mapping results are very similar to those in [10] in visual perception, but the number of Steiner vertices added by our approach is much fewer than [10], as shown in Table 1. In this table, we show the numbers of Steiner vertices introduced by both methods before and after removing redundant Steiner vertices.

\subsection{Discussion with Related Work}

For a 3D morphing application, Zöckler et al. [26] used edge-swaps to solve foldovers on base mesh, also called warp mesh. There are many significant differences between our algorithm and theirs. First, each edge of their warp mesh is a direct line connecting two features on the embedding. In contrast, we need to compute a path on the embedding for each edge of warp mesh. We solve foldovers by edge swaps, and each edge swap on the base mesh corresponds to a path swap on the embedding. Second, their approach is not robust and cannot avoid foldovers, as shown in Fig. 10. In contrast, we present a theoretically robust algorithm to detect and solve foldovers. Moreover, our algorithm can robustly handle parameterization under a large set of constraints. These constraints can be very distinct in their positions.

For the path matching-based algorithm [10], [11], [19], [21], an undesired "swirl" situation may arise. In [19], swirling indicates that the matching path may wind around the other path. From our observation, this usually happens while the neighbor vertex of the matching path is located at 


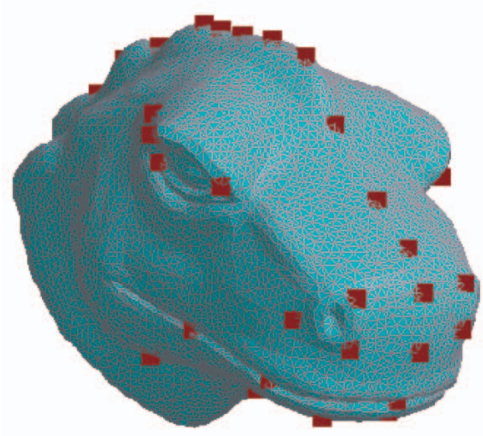

(a)

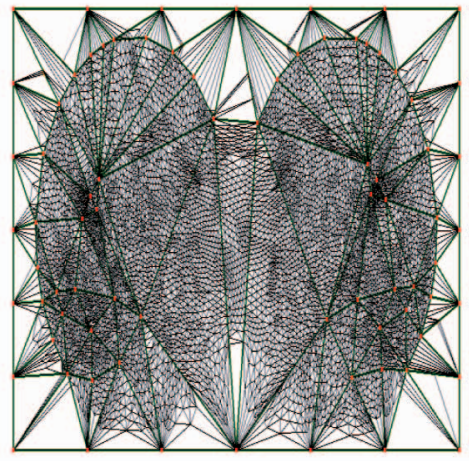

(d)

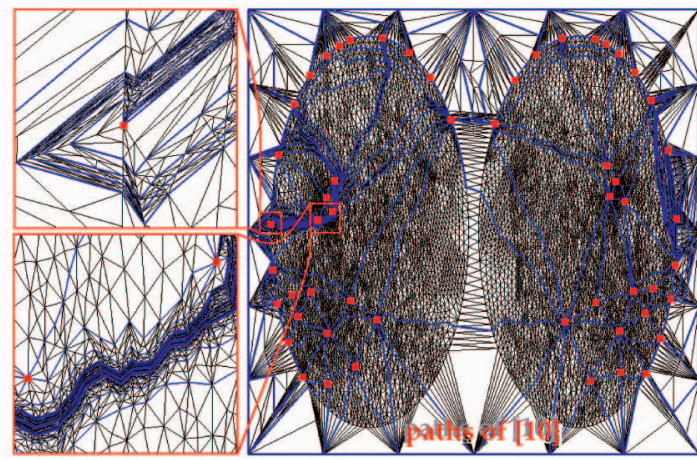

(g)

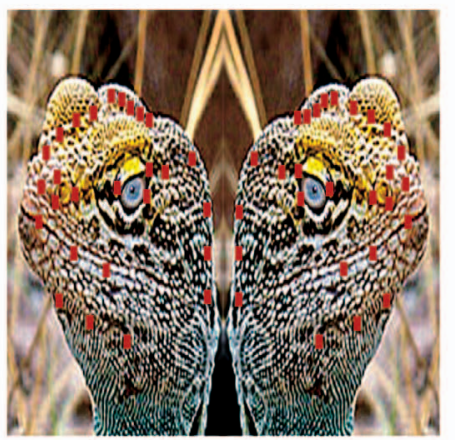

(b)

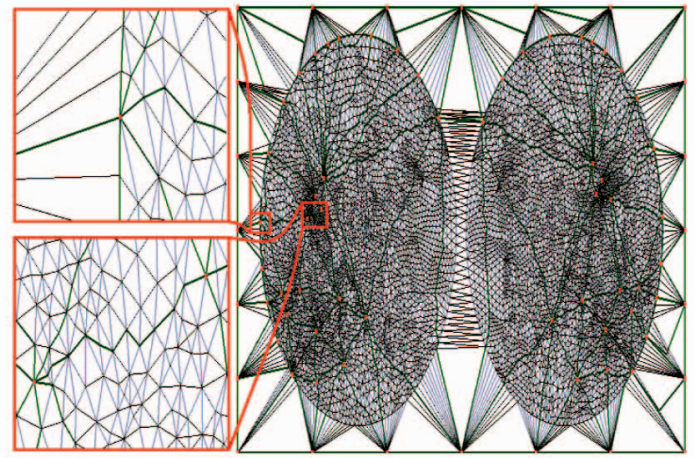

(c)

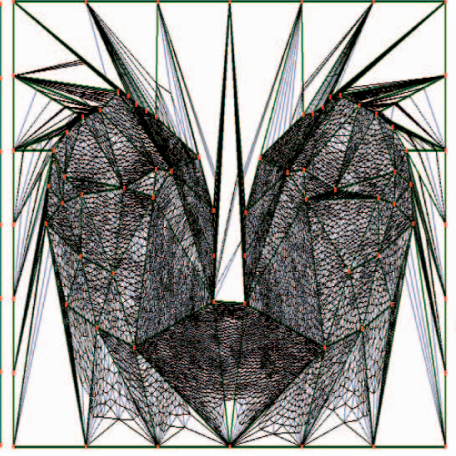

(e)

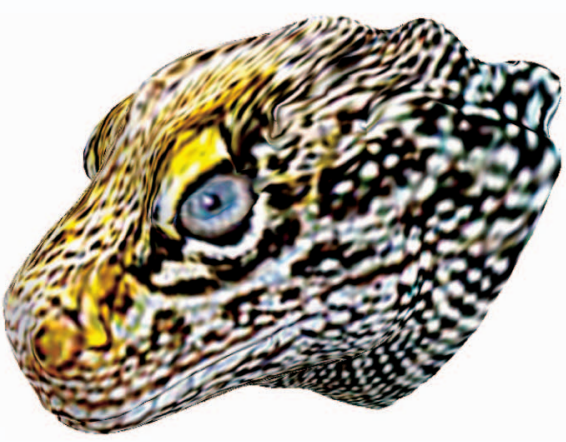

(f)

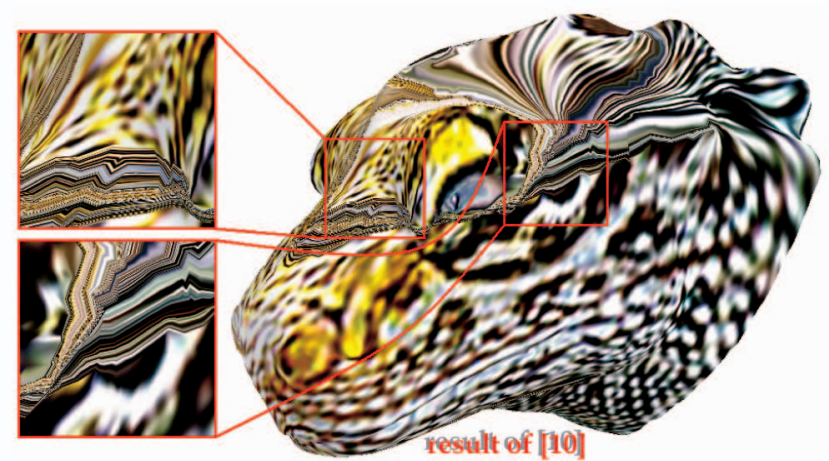

(h)

Fig. 14. (a) Input mesh $M$. (b) Input texture. (c) The embedding $P^{M+}$ of $M$ and the initial paths (green) of base mesh $B^{M+}$. (d) Initial base mesh $B^{M+}$. (e) $B^{M+}$ after feature matching. (f) Texture mapping result after smoothing from (e). (g) The path matching using that in [10]. (h) Texture mapping result in [10].

the different side between the embedding domain and the texture. Our warping strategy can easily deal with this issue using the edge swap. We will illustrate this by a simple example, as shown in Fig. 11. Let $A, B$, and $C$ be the three feature vertices, where there exists a matching path $A B$, such that the feature point $C$ is at the different side between $P^{M+}$ and the texture. The feature vertex $C$ should move around $A$ or $B$ to align the destination on $P^{M+}$ since the existence of path $A B$. In contrast, using our approach, this feature vertex $C$ can move directly to the destination using the edge swap of path $A B$. The result of matching paths using [10] is shown in Fig. 11d. In addition, Praun et al. [19] and Schreiner et al. [21] try to avoid swirling using the heuristics that delays such bad path matching or reroutes the path to let neighbor features be at the same side between the embedding and the texture. In contrast, our approach simply uses the edge swap to avoid swirling instead of these more complicated heuristics. Finally, regarding a path finding, we should also mention the following observation. For [10], [11], [19], and [21], their path-matching algorithms require tedious tests for path blocking and path searching in a global and greedy manner. In contrast, the path finding and block checking are only executed within the local enclosing quadrilateral, as shown in the warping algorithm of Section 3.2.2.

\section{Conclusion}

We presented a new and theoretically robust warping algorithm to align features for the planar embedding of a triangle mesh. Our algorithm robustly detects where a foldover occurs and determines where to execute edge swaps. This new algorithm is extended to solve texture 

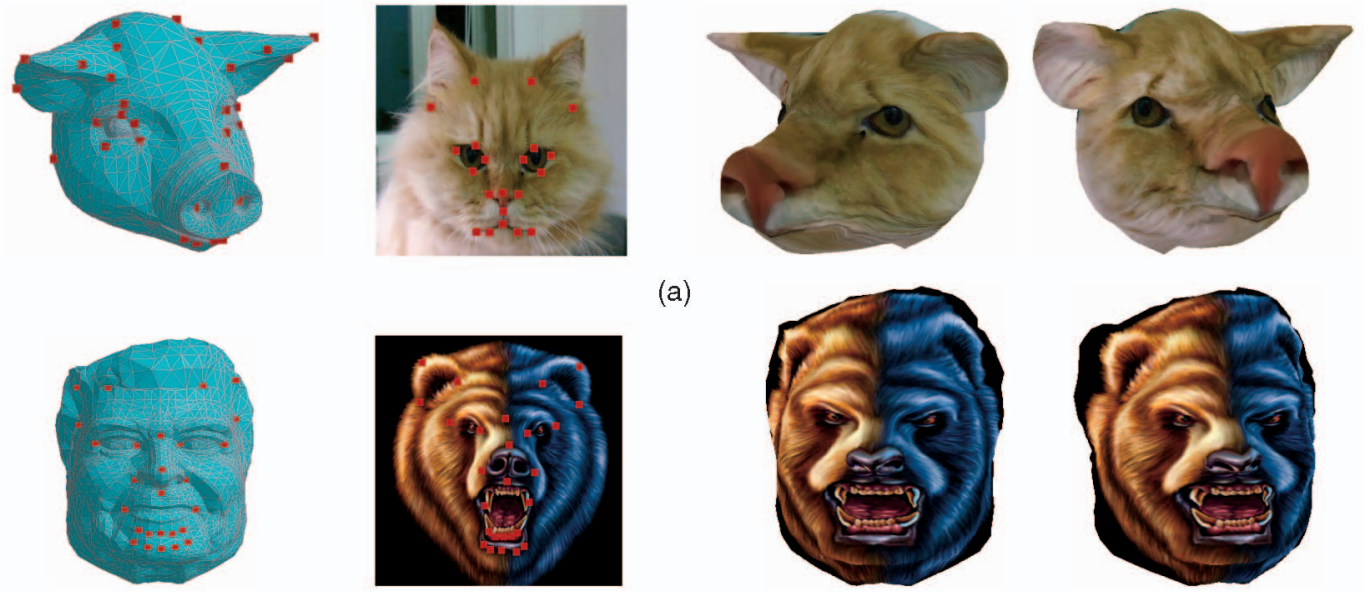

(b)
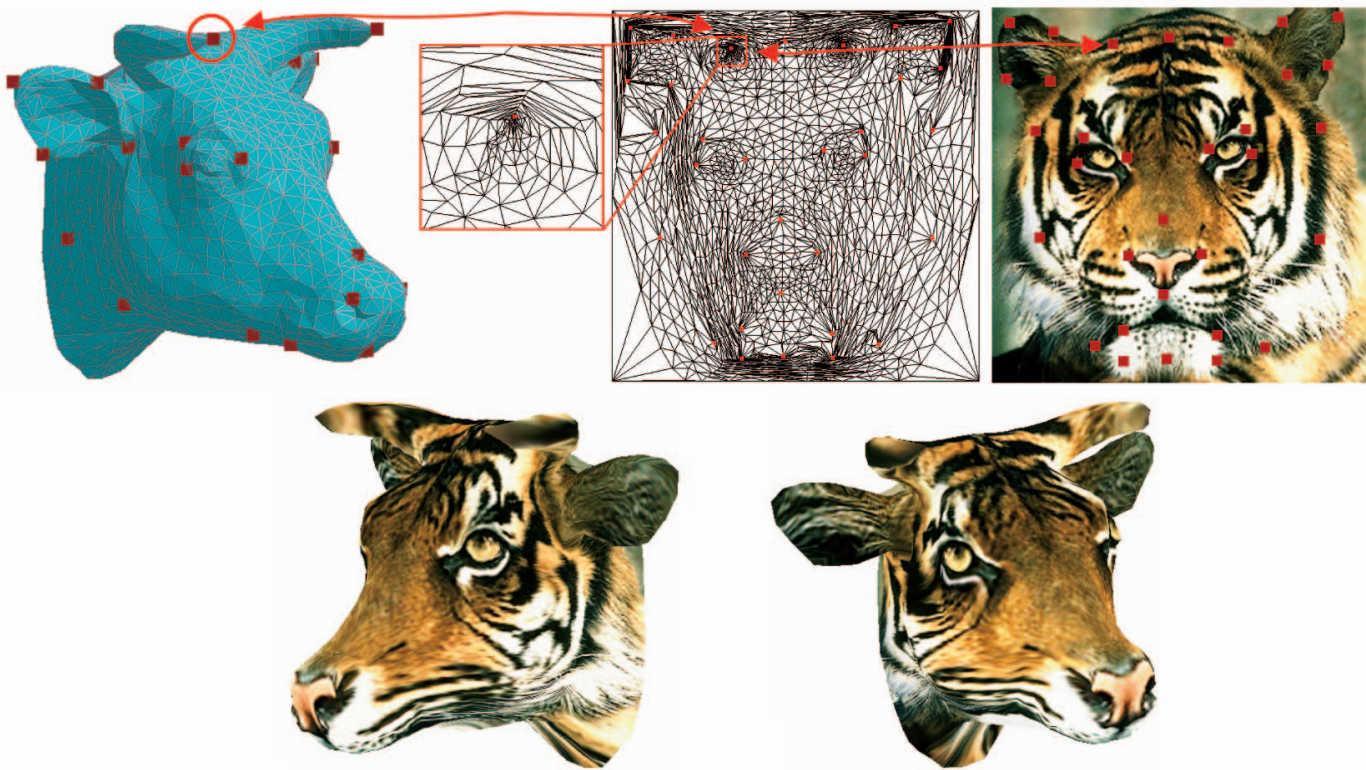

(c)
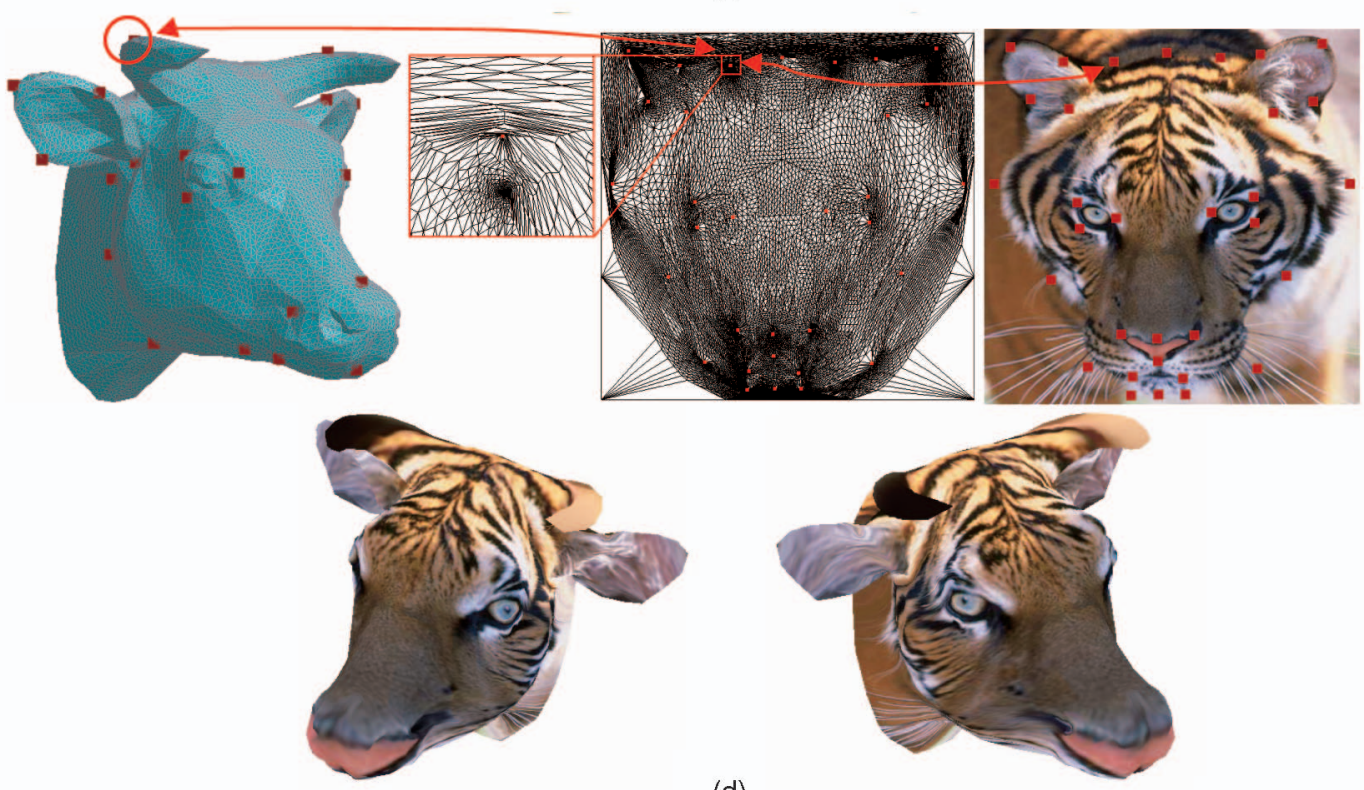

(d)

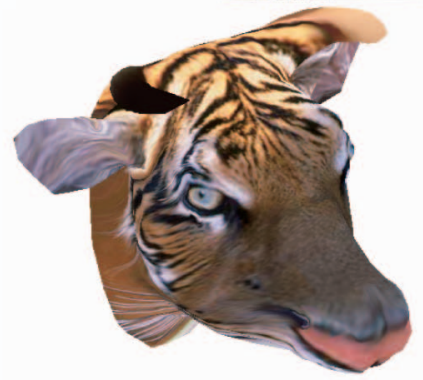

Fig. 15. More results. In (c) and (d), the embeddings are the final results after smoothing. 

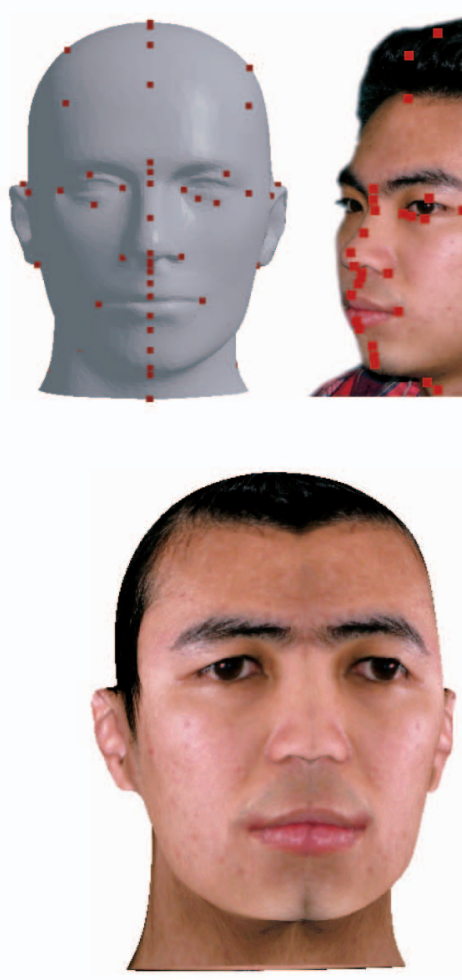

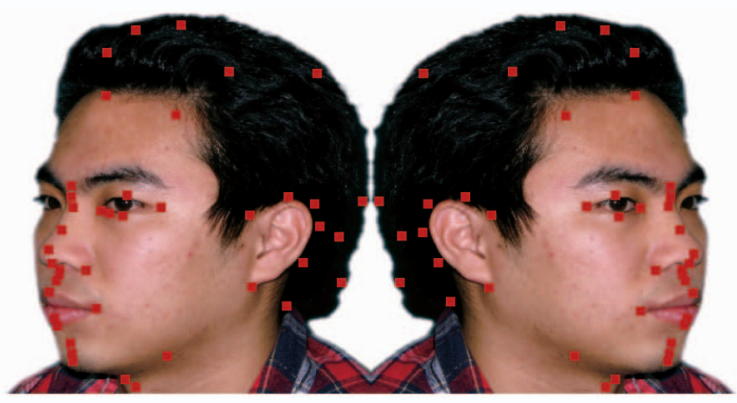

(a)

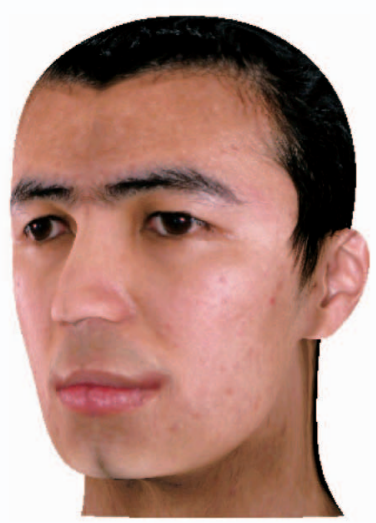

(b)

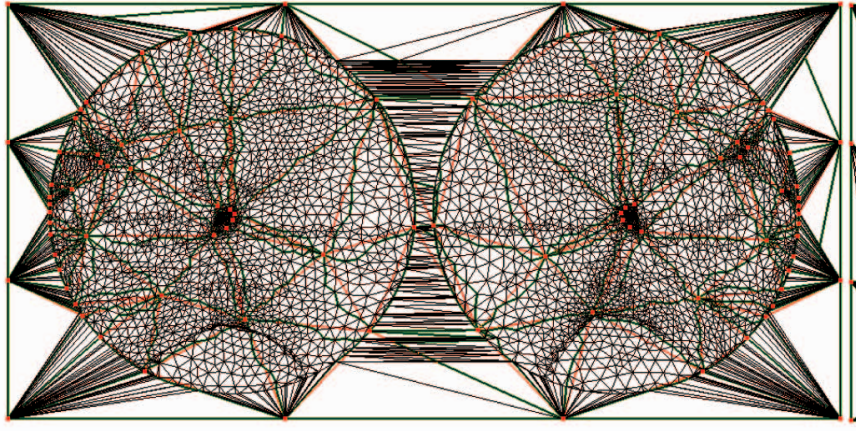

(c)

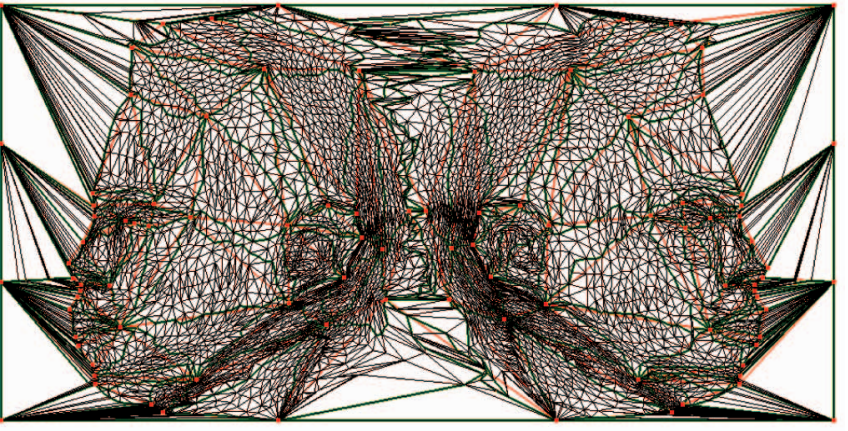

(d)

Fig. 16. (a) A hairless head model with the texture image. (b) Texture mapping results. (c) Initial parametric map. (d) Final embedding after mesh smoothing.

mapping with hard constraints. This new algorithm employs path swaps to eliminate possible triangle foldovers during the matching constrained vertices process between embedding and texture. We experimentally verify the proposed method and also compare it with the state-ofart method for some challenging examples. Our results show the advantage of the proposed method in handling challenging constraints well. In the near future, many related and interesting researches will be continued. For example, minimizing the number of edge swaps and intelligently scheduling edge-swaps to avoid skinny triangle shapes of the base mesh will pose very challenging problems. In addition, we plan to apply our method to other applications, such as morphing and consistent parameterization of several models. Our smoothing is an iterative and local relaxation scheme. It cannot be computed on the fly. We will investigate possible alternative to fast smoothing embedding. In constrained texture mapping, constraints might be localized, and therefore, they create a sort of groups that might cause a highly distorted area. A global relaxation method may reduce the problem (at least visually). We will investigate this possibility in the near future.

\section{ACKNOWLEDGMENTS}

The authors give their special thanks to Dr. Kun Zhou and Jianwei Han at Microsoft Research Asia who implemented a matchmaker program and helped them with the experimental comparison to that in [10]. They also give their special thanks to their colleague Professor Sun-Yuan Hsieh, who helped them check the correctness of proof in this paper. This project is supported by the National Science Council, Taiwan, under Contracts NSC95-2221-E-006-368, NSC-95-2221-E-006-193-MY2, and NSC96-2628-E-006-200-MY3. 

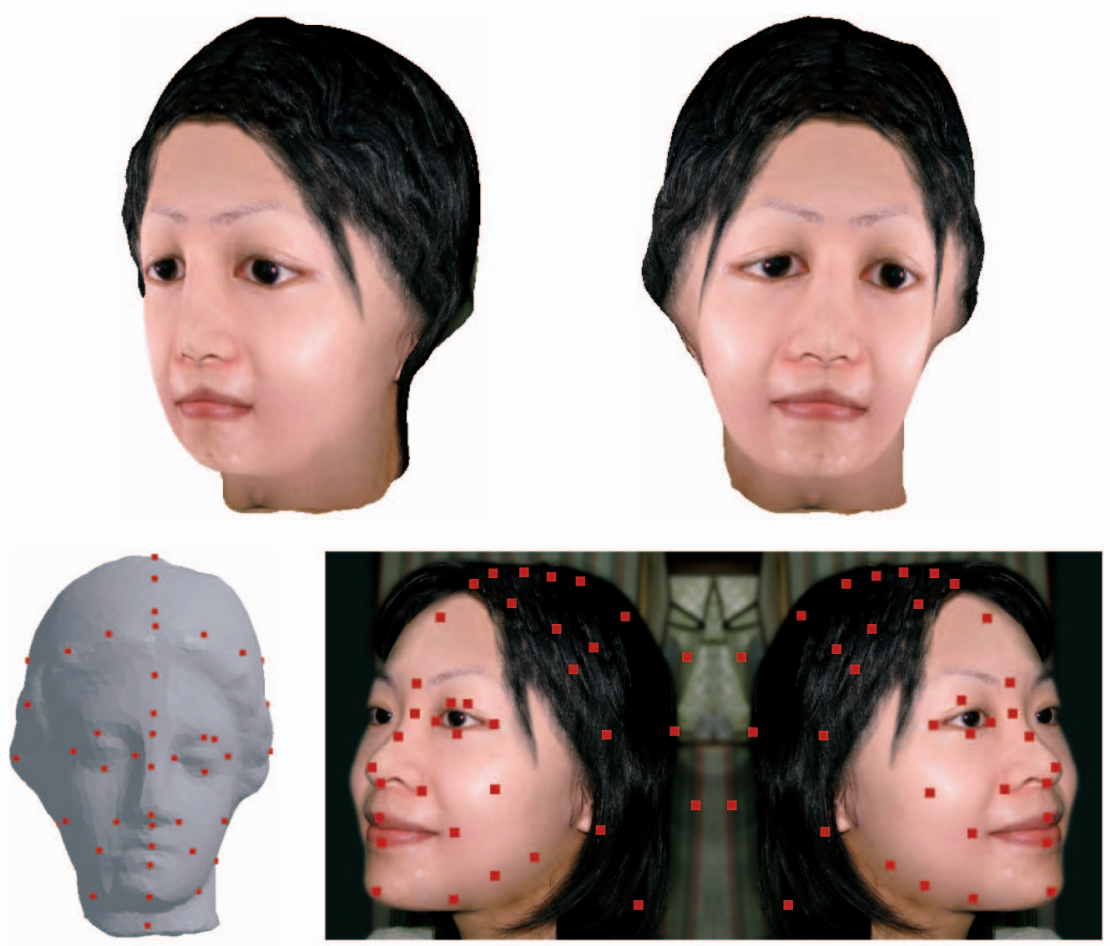

Fig. 17. An Igea model and the texture mapping results.

TABLE 1

The Statistics for the Texture Mapping Samples

\begin{tabular}{|c|c|c|c|c|c|c|c|c|}
\hline Model & \# vertices & $\begin{array}{c}\text { \# trian- } \\
\text { gles }\end{array}$ & $\begin{array}{c}\text { \# feature } \\
\text { points }\end{array}$ & $\begin{array}{c}\text { Feature } \\
\text { warping } \\
\text { (seconds) }\end{array}$ & $\begin{array}{c}\text { \# Steiner } \\
\text { (initial) }\end{array}$ & $\begin{array}{c}\text { \# Steiner } \\
\text { (final) }\end{array}$ & $\begin{array}{c}\text { \# Steiner } \\
\text { (initial) } \\
{[10]}\end{array}$ & $\begin{array}{c}\text { \# Steiner } \\
\text { (final) } \\
{[10]}\end{array}$ \\
\hline Fig. 7 & 1770 & 3526 & 24 & 0.625 & 77 & 7 & 596 & 43 \\
\hline Fig. 13 & 1808 & 3602 & 25 & 1.712 & 128 & 10 & 1488 & 368 \\
\hline Fig. 14 & 10017 & 20008 & 54 & 10.641 & 94 & 34 & 9365 & 2687 \\
\hline Fig. 15(a) & 1772 & 3450 & 21 & 3.359 & 383 & 21 & 1343 & 154 \\
\hline Fig. 15(b) & 1657 & 3300 & 27 & 0.75 & 69 & 2 & 456 & 51 \\
\hline Fig. 15(c) & 1697 & 3384 & 32 & 4.39 & 223 & 64 & 1318 & 193 \\
\hline Fig. 15(d) & 10736 & 21404 & 32 & 14.5 & 127 & 8 & 1358 & 133 \\
\hline Fig. 16 & 5184 & 10354 & 83 & 10.86 & 183 & 38 & 2620 & 361 \\
\hline Fig. 17 & 4149 & 8284 & 71 & 6.14 & 122 & 25 & 1425 & 192 \\
\hline
\end{tabular}

\#Steiner (initial) and \#Steiner (final) are the number of Steiner vertices before and after removing.

\section{REFERENCES}

[1] M. Alexa, "Merging Polyhedral Shapes with Scattered Features," The Visual Computer, vol. 16, pp. 26-37, 2000.

[2] M. Desbrun, M. Meyer, and P. Alliez, "Intrinsic Parameterizations of Surface Meshes," Proc. Eurographics '02/Computer Graphics Forum, vol. 21, no. 3, pp. 209-218, 2002.

[3] M. Eck, T. Derose, T. Duchamp, H. Hoppe, M. Lounsbery, and W. Stuetzle, "Multiresolution Analysis of Arbitrary Meshes," Proc. ACM SIGGRAPH' '95, pp. 173-182, 1995.

[4] I. Eckstein, V. Surazhsky, and C. Gotsman, "Texture Mapping with Hard Constraints," Proc. Eurographics '01/Computer Graphics Forum, vol. 20, no. 3, pp. 95-104, 2001.

[5] M.S. Floater, "Parameterization and Smooth Approximation of Surface Triangulations," Computer Aided Geometric Design, vol. 14, no. 3, pp. 231-250, 1997.

[6] C.-H. Lin and T.-Y. Lee, "Metamorphosis of 3D Polyhedral Models Using Progressive Connectivity Transformations," IEEE Trans. Visualization and Computer Graphics, vol. 11, no. 1, pp. 2-12, Jan./Feb. 2005.
[7] K. Fujimura and M. Makarov, "Foldover-Free Image Warping," Graphical Models and Image Processing, vol. 60, no. 2, pp. 100-111, 1998.

[8] C. Gotsman, X. Gu, and A. Sheffer, "Fundamentals of Spherical Parameterization for 3D Meshes," ACM Trans. Graphics, vol. 22, no. 3, pp. 358-363, 2003.

[9] K. Hormann and G. Greiner, "MIPS: An Efficient Global Parametrization Method," Proc. Curve and Surface Design Conf., pp. 153-162, 1999.

[10] V. Kraevoy, A. Sheffer, and C. Gotsman, "Matchmaker: Constructing Constrained Texture Maps," ACM Trans. Graphics, vol. 22, no. 3, pp. 326-333, 2003.

[11] V. Kraevoy and A. Sheffer, "Cross-Parameterization and Compatible Remeshing of 3D Models," ACM Trans. Graphics, vol. 23, no. 3, pp. 861-869, 2004.

[12] B. Lévy, "Constrained Texture Mapping for Polygonal Meshes," Proc. ACM SIGGRAPH '01, pp. 417-424, 2001.

[13] B. Lévy, S. Petitjean, N. Ray, and J. Maillot, "Least Squares Conformal Maps for Automatic Texture Atlas Generation," ACM Trans. Graphics, vol. 21, no. 3, pp. 362-371, 2002. 
[14] T.-Y. Lee and P.H. Huang, "Fast and Institutive Polyhedra Morphing Using SMCC Mesh Merging Scheme," IEEE Trans. Visualization and Computer Graphics, vol. 9, no. 1, pp. 85-98, Jan.Mar. 2003.

[15] T.-Y. Lee, C.-Y. Yao, H.-K. Chu, M.-J. Tai, and C.-C. Chen, "Generating Genus-n-to-m Mesh Morphing Using Spherical Parameterization: Research Articles," Computer Animation and Virtual Worlds, vol. 17, no. 3, pp. 433-443, 2006.

[16] C.-H. Lin, T.-Y. Lee, H.-K. Chu, and Z.-Y. Yao, "Progressive Mesh Metamorphosis," J. Computer Animation and Virtual Worlds, vol. 16, nos. 3-4, pp. 487-498, 2005

[17] J. Maillot, H. Yahia, and A. Verroust, "Interactive Texture Mapping," Proc. ACM SIGGRAPH '93, pp. 27-34, 1993.

[18] U. Pinkall and K. Polthier, "Computing Discrete Minimal Surfaces and Their Conjugates," Experimental Math., vol. 2, no. 1, pp. 15-36, 1993.

[19] E. Praun, W. Sweldens, and P. Schröder, "Consistent Mesh Parameterizations," Proc. ACM SIGGRAPH '01, pp. 179-184, 2001.

[20] P.V. Sander, J. Snyder, S.J. Gortler, and H. Hoppe, "Texture Mapping Progressive Meshes," Proc. ACM SIGGRAPH '01, pp. 409-416, 2001.

[21] J. Schreiner, A. Asirvatham, E. Praun, and H. Hoppe, "InterSurface Mapping," ACM Trans. Graphics, vol. 23, no. 3, pp. 870$877,2004$.

[22] J.R. Shewchuk, "Triangle: Engineering a 2D Quality Mesh Generator and Delaunay Triangulator," Lecture Notes in Computer Science, vol. 1148, pp. 203-222, 1996.

[23] O. Sorkine, D. Cohen-Or, R. Goldenthal, and D. Lischinski, "Bounded-Distortion Piecewise Mesh Parameterization," Proc. IEEE Visualization, pp. 355-362, 2002.

[24] W.T. Tutte, "Convex Representations of Graphs," Proc. London Math Soc., vol. 10, pp. 304-320, 1960.

[25] K. Zhou, X. Wang, Y. Tong, M. Desbrun, B. Guo, and H.-Y. Shum, "TextureMontage: Seamless Texturing of Arbitrary Surfaces from Multiple Images," ACM Trans. Graphics, vol. 24, no. 3, pp. 11481155,2005

[26] M. Zöckler, D. Stalling, and H.-C. Hege, "Fast and Intuitive Generation of Geometric Shape Transitions," The Visual Computer, vol. 16, no. 5, pp. 241-253, 2000.

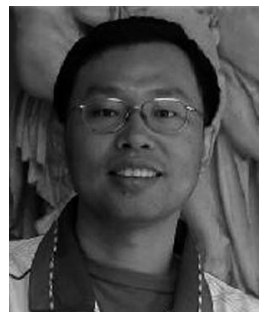

Tong-Yee Lee received the $\mathrm{PhD}$ degree in computer engineering from Washington State University, Pullman, in May 1995. He is currently a professor in the Department of Computer Science and Information Engineering, National Cheng-Kung University, Tainan, Taiwan. He leads a Computer Graphics Group/Visual System Lab, National Cheng-Kung University (http:// graphics.csie.ncku.edu.tw/). His current research interests include computer graphics, nonphotorealistic rendering, image-based rendering, visualization, virtual reality, surgical simulation, medical visualization and medical system, and distributed and collaborative virtual environment. He serves as an associate editor for IEEE Transactions on Information Technology in Biomedicine from 2000 to 2007 . He is also on the editorial advisory board of the Journal Recent Patents on Engineering, an editor of the Journal of Information Science and Engineering, and a region editor of the Journal of Software Engineering. He is a member of the IEEE and ACM.

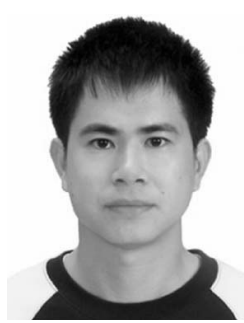

Shao-Wei Yen received the BS degree from the Department of Civil Engineering, National Taiwan University, Taipei, in 2000 . He is currently working toward the PhD degree at the Department of Computer Science and Information Engineering, National Cheng-Kung University. His research interests include computer graphics and mesh parameterization.

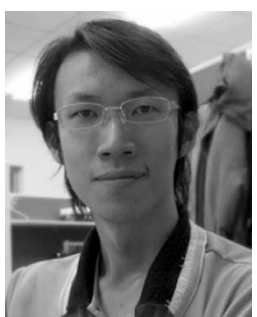

I-Cheng Yeh received the BS degree from the Department of Computer and Information Science, National Chiao Tung University, Taiwan, in 2004. He is currently working toward the $\mathrm{PhD}$ degree at the Department of Computer Science and Information Engineering, National Cheng-Kung University. His research interests include computer graphics and mesh parameterization.

$\triangleright$ For more information on this or any other computing topic, please visit our Digital Library at www.computer.org/publications/dlib. 